\title{
ARTíCULO
}

\section{Biota del intermareal rocoso expuesto de la Isla Grande de Chiloé, Archipiélago de Chiloé, Chile: Patrones de diversidad e implicancias ecológicas y biogeográficas}

Exposed rocky intertidal biota of the Isla Grande de Chiloé, Archipiélago de Chiloé, Chile: Diversity patterns and ecological and biogeographic implications

\section{Carlos Velásquez ${ }^{1}$, Eduardo Jaramillo², Patricio A. Camus ${ }^{3,4}$, Mario Manzano ${ }^{5}$ y Roland Sánchez ${ }^{6}$}

${ }^{1}$ Programa Magíster en Ciencias Mención Recursos Hídricos, Escuela de Graduados, Facultad de Ciencias, Universidad Austral de Chile, Valdivia, Chile. cfvelasque@gmail.com

${ }^{2}$ Facultad de Ciencias, Universidad Austral de Chile, Casilla 567, Valdivia, Chile

${ }^{3}$ Departamento de Ecología, Facultad de Ciencias, Universidad Católica de la Santísima Concepción, Casilla 297, Concepción, Chile ${ }^{4}$ Centro de Investigación en Biodiversidad y Ambientes Sustentables (CIBAS), Universidad Católica de la Santísima Concepción, Concepción, Chile

${ }^{5}$ Programa Doctorado en Biología Marina, Escuela de Graduados, Facultad de Ciencias, Universidad Austral de Chile, Valdivia, Chile ${ }^{6}$ Programa Doctorado en Ciencias Mención Ecología y Evolución, Escuela de Graduados, Facultad de Ciencias, Universidad Austral de Chile, Valdivia, Chile

\begin{abstract}
The northern part of the Chiloé Island $\left(41.8^{\circ} \mathrm{S}\right)$ has been linked to an abrupt biogeographic break in the Southeastern Pacific; a break which represents the northern limit of the Magellanic Province, a large zone geographically characterized by a broken coastline as opposed to the Chilean coast further north. This study represents a first eco-biogeographical characterization of the rocky intertidal macrobiota along the northwest coast of Chiloé Island, a coastal line stretching nearly $50 \mathrm{~km}$. Sampling was carried out at 5 sites using the same systematic design (transects perpendicular to the coast using $0.25 \mathrm{~m}^{2}$ quadrats in 4 tidal levels), and the data were analyzed by using multivariate statistical methods. A total of 38 species were recorded, with a consistent zonation and dominance across sites, albeit minor differences due to local variations in abundance and cover of mobile and sessile species. Virtually all taxa were organisms widely distributed along the coast of Chile, showing patterns of community structure and composition similar to those documented for central Chile. We reinforce the need to reassess the biogeographic character of the coasts directly exposed to the Pacific in the austral zone of Chile, and the role of historical processes on the northwest coast of Chiloé having in mind that the study area was not affected by glacial advances in the late Pleistocene.
\end{abstract}

Key words: Glaciation, Magellan Province, biogeographic transition, zonation

Resumen.- La parte norte de la Isla Grande de Chiloé $\left(41,8^{\circ} \mathrm{S}\right)$ ha sido vinculada a un quiebre biogeográfico abrupto en el Pacífico Sudeste, lo que marca el límite norte de la Provincia Magallánica, una gran zona geográfica caracterizada por tener una costa desmembrada en oposición a la fisonomía de la costa Chilena ubicada más al norte. Este estudio presenta una primera caracterización ecológica y biogeográfica de la macrobiota intermareal rocosa de la zona noroeste de la Isla Grande de Chiloé, una línea costera de aproximadamente $50 \mathrm{~km}$. En 5 sitios se aplicó un muestreo sistemático (transectos perpendiculares a la costa usando cuadrantes de $0,25 \mathrm{~m}^{2}$ en 4 niveles mareales), analizando los datos mediante métodos multivariados. Se registró un total de 38 especies cuya zonación y dominancia fueron consistentes entre sitios, aunque con diferencias menores debidas a variaciones locales en abundancia y cobertura de especies particulares. Prácticamente todos los taxa fueron organismos comunes de amplia distribución en Chile, mostrando patrones comunitarios de composición y estructura equivalentes a los documentados para la costa de Chile central. Se discute la necesidad de reevaluar el carácter biogeográfico de las costas expuestas al Pacífico en la zona austral de Chile, y el rol de los procesos históricos en la costa noroeste de Chiloé considerando que el área de estudio no fue afectada por los avances glaciares a fines del Pleistoceno.

Palabras clave: Glaciación, Provincia Magallánica, transición biogeográfica, zonación 


\section{INTRODUCCIÓN}

Una tarea central de la biogeografía es el reconocimiento de estructuras anidadas de unidades espaciales de distinta jerarquía (regiones, provincias, distritos, y otros) en función de sus diferencias en composición biótica (e.g., véase Ferro \& Morrone 2014). Los límites entre las unidades detectadas se asocian habitualmente a la existencia de quiebres en la distribución de conjuntos de especies, debido al efecto de barreras fisiológicas o ecológicas que impiden o restringen la dispersión, o de filtros que permiten el paso selectivo de especies (Brown \& Lomolino 1998). En algunos casos, sin embargo, los límites entre unidades biogeográficas no son discretos o absolutos y pueden adoptar la forma de una zona de transición, es decir un área de superposición biótica promovida por cambios históricos y ecológicos que permiten la mezcla de taxa pertenecientes a diferentes componentes bióticos (Ferro \& Morrone 2014). Un ejemplo notable de estos procesos se encuentra en la costa sur de Chile, donde la Isla Grande de Chiloé $\left(\sim 42^{\circ} \mathrm{S}\right)$ es el hito geográfico que marca una de las mayores transiciones bióticas en el Pacífico oriental, cuyas determinantes históricas y ecológicas aún no son adecuadamente conocidas.
El rasgo más distintivo en la geomorfología costera de Chile es su diferenciación latitudinal en 2 segmentos discretos de mínima y máxima complejidad topográfica a gran escala, separados por el Canal de Chacao (Fig. 1): (a) una costa continua y virtualmente recta desde Arica $\left(18,4^{\circ} \mathrm{S}\right)$ hasta Carelmapu $\left(41,7^{\circ} \mathrm{S}\right)$; y (b) una costa totalmente fragmentada desde el extremo norte de la Isla Grande de Chiloé $\left(41,8^{\circ} \mathrm{S}\right)$ al Cabo de Hornos $\left(56^{\circ} \mathrm{S}\right)$, con un paisaje complejo formado por archipiélagos, fiordos y canales interiores protegidos de características principalmente estuariales (Viviani 1979, Lancellotti \& Vásquez 2000, Camus 2001), que se habrían originado por la acción combinada de eventos tectónicos y avances y retrocesos glaciales en el Pleistoceno hasta hace 20,000 años (e.g., Villagrán 1990, Heusser et al. 1992, Abarzúa et al. 2004, Villagrán et al. 2004). En este escenario, la Isla Grande de Chiloé (en adelante IG-Chiloé), la mayor del Archipiélago de Chiloé (ca., $8.400 \mathrm{~km}^{2}$ ) y la segunda mayor del territorio chileno (después de Isla Grande de Tierra del Fuego), tiene especial importancia biológica debido a su tamaño, historia evolutiva y ubicación geográfica.

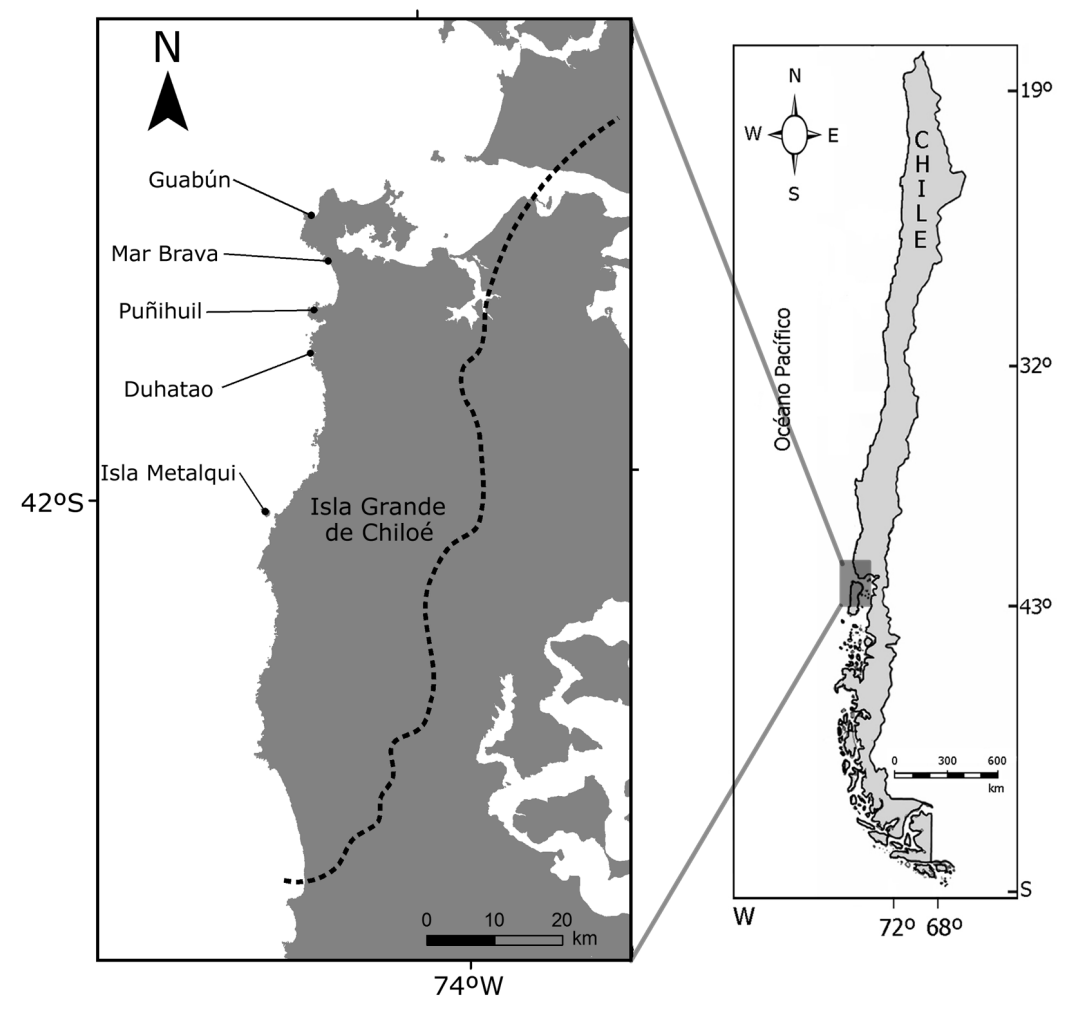

Figura 1. Ubicación de los sitios de estudio en la costa noroeste de la Isla Grande de Chiloé, sur de Chile. La línea segmentada (basada en Latorre et al. 2007) indica el límite oeste del avance de los glaciares durante el Último Máximo Glacial ca., 20,000 años AP / Location of the study sites along the northwestern coast of the Isla Grande de Chiloé, southern Chile. The dashed line (after Latorre et al. 2007) indicates the westernmost limit of glacier advance during the Last Glacial Maximum ca., 20.000 years BP 
La IG-Chiloé es parte de uno de los hotspots terrestres de biodiversidad con mayor concentración de endemismo en el planeta (Myers et al. 2000), y alto grado de amenaza por el impacto histórico de diversas actividades antrópicas (e.g., Armesto et al. 1998, Simonetti 1999, Arroyo et al. 2008). En el ambiente marino, la IG-Chiloé marca tanto el término de una zona de transición biogeográfica a partir del límite sur de la provincia Peruana $\left(30-33^{\circ} \mathrm{S}\right)$, como el inicio de la provincia Magallánica templada-fría que se extiende hasta el Cabo de Hornos. Además, la IG-Chiloé es el área donde la Corriente Circumpolar Antártica alcanza la costa de Sudamérica y se divide en dos ramas, formando el sistema de Corrientes de Humboldt hacia el norte y la Corriente del Cabo de Hornos hacia el sur (véase revisiones de Fernández et al. 2000, Camus 2001, Thiel et al. 2007). El origen de esta configuración físicabiológica deriva del proceso de quiebre del supercontinente Mesozoico de Gondwana (e.g., McLoughlin 2001), formado por la mayoría de las actuales masas de tierra del hemisferio sur, y en particular de la separación entre América del Sur y la Antártica durante el Terciario (Oligoceno, hace ca., $30 \mathrm{Ma}$ ) que llevó al establecimiento del sistema frío de Humboldt y de la biota austral ancestral (e.g., Crisci et al. 1991, Camus 2001). El quiebre de Gondwana explica a su vez la afinidad biogeográfica reconocida por diversos autores entre las biotas marinas de la zona austral de Chile, Australia, Nueva Zelanda, Tasmania, Antártica e islas subantárticas (e.g., Hedgpeth 1969, Alveal et al. 1973, Larraín 1975, Viviani 1979, Santelices 1980, Jaramillo 1981, Castilla et al. 1993, Moyano 1996, Cárdenas et al. 2008).

La importancia biogeográfica de la biota bentónica de la IG-Chiloé ha sido destacada en numerosos estudios comparativos del litoral rocoso chileno (e.g., Balech 1954, Knox 1960, Etcheverry 1964, Stuardo 1964, Viviani 1979, Santelices 1980, Moyano 1983, Brattström \& Johanssen 1983, Brattström 1990, Fernández et al. 2000, Lancellotti \& Vásquez 2000, Camus 2001). Sin embargo el conocimiento biológico de la costa oeste expuesta al Pacífico de la IG-Chiloé es escaso o impreciso, y continúa limitadobásicamente a la fauna de playas de arena (e.g., Jaramillo et al. 2000, Dugan et al. 2004). De hecho la información disponible se basa en parte en descripciones que no registran localidades específicas (e.g., Knox 1960), y en su mayoría proviene de expediciones científicas Suecas e Inglesas realizadas hace más de medio siglo, destacando la Lund University Chile Expedition 1948-49 (e.g., Mortensen 1952, Menzies 1962, Brattström 1990) y la Royal Society of London Expedition to Southern Chile 1958-59 (e.g., Pawson 1964, Pawson 1966, Dell 1971), a las que sólo recientemente se sumaron las campañas chilenas del Programa CIMAR Fiordos (e.g., Cárdenas et al. 2008, Ramajo \& Osorio
2010). No obstante, casi todos estos estudios se han enfocado en la biota de fondos blandos, y se han concentrado en la zona del mar interior que presenta una salinidad y exposición al oleaje mucho más bajas que las de las costas abiertas al Pacífico, y además difiere significativamente en otras características físicoquímicas así como en sus cargas y dinámicas de transporte de sedimentos y nutrientes (e.g., Pickard 1971, Silva et al. 1997, Ahumada et al. 2000, Dávila et al. 2002, Haussermann \& Forsterra 2009). En este marco, determinar si los regímenes físicos contrastantes entre las costas este y oeste del Archipiélago de Chiloé ejercen un diferencial sobre la biota de costas rocosas es un paso necesario para clarificar la naturaleza de la transición biogeográfica de los $42^{\circ} \mathrm{S}$.

Por lo tanto, el presente estudio tuvo como objetivo primario proveer una primera caracterización de la diversidad de la biota intermareal rocosa de la costa oeste de la IG-Chiloé, a fin de establecer su carácter biogeográfico. Si bien la importancia de las diferencias este-oeste sobre la biogeografía del área de Chiloé fue señalada tempranamente por varios autores (e.g., Dahl 1960, Castilla 1979, Viviani 1979), sus efectos aún no han sido aclarados debido a la falta de estudios sistemáticos de diversidad. A esto se agrega el efecto histórico de los eventos geológicos y glaciales del Cuaternario sobre la oceanografía, nivel del mar y geomorfología costera en el sur de Chile (e.g., Clapperton 1994, Latorre 2008, Hervé et al. 2009), que son un factor clave para comprender los patrones biogeográficos marinos actuales (Ortlieb et al. 1994, Ortlieb 1995) cuya importancia no ha sido considerada adecuadamente. De hecho estudios recientes (e.g., Hulton et al. 2002, Villagrán \& Armesto 2005, Latorre et al. 2007, Glasser et al. 2008) revelan que durante el último máximo glacial los glaciares no avanzaron sobre la costa norte (desde $\sim 73,5^{\circ} \mathrm{O}$ ) y gran parte de la costa oeste $\left(\sim 41,7-42,7^{\circ} \mathrm{S}\right)$ de la IG-Chiloé, pero en contraste tuvieron un gran impacto en toda su costa este y la zona del mar interior (Fig. 1). Si bien el efecto biogeográfico de esta glaciación diferencial no ha sido evaluado, la costa Pacífica de la IG-Chiloé -pese al virtual desconocimiento de su biota- es sin duda un área de alta significancia biológica, por locual resulta afortunado que albergue importantes áreas de conservación marina como el Monumento Natural Islotes de Puñihuil y el sector norte del Parque Nacional Chiloé (Rovira et al. 2008).

En un contexto general, la literatura muestra que los gradientes de exposición al oleaje afectan fuertemente la distribución y composición de la biota intermareal en costas rocosas, por lo cual la diversidad y estructura de estas comunidades a escala biogeográfica dependería a un grado importante de la variación espacial en exposición (e.g., véase Smith \& Simpson 2002, y revisiones de Bustamante \& Branch 1996, Ricciardi \& Bourget 1999). Por lo tanto el patrón 
latitudinal de reemplazo de especies asociado a la transición biogeográfica de los $42^{\circ} \mathrm{S}$ podría estar modulado por el fuerte gradiente longitudinal de exposición en la misma área. Esta predicción es examinada indirectamente en el presente estudio evaluando los patrones de riqueza, abundancia, composición y zonación de las especies sésiles y móviles que forman la comunidad intermareal rocosa de la costa expuesta al Pacífico de la Isla Grande de Chiloé, en 5 sitios representativos de la variación fisiográfica observada a través de $\sim 50 \mathrm{~km}$ de costa. Esta información es analizada comparativamente para determinar la afinidad biogeográfica de la costa oeste de la IGChiloé con los ambientes protegidos del mar interior y la costa no fragmentada al norte de los $42^{\circ} \mathrm{S}$, y proveer información que permita establecer criterios de gestión para la conservación de su biodiversidad.

\section{Materiales Y MÉTODOS}

\section{Área de ESTUdio y MUESTREOS}

El estudio se realizó en el hábitat intermareal rocoso del sector oeste de la Isla Grande de Chiloé, en 5 sitios abarcando 50 km de costa (Fig. 1): Guabún (41 $\left.48^{\circ} \mathrm{S}, 7^{\circ} 01^{\prime} \mathrm{O}\right)$, Mar Brava ( $\left.41^{\circ} 55^{\prime} \mathrm{S}, 74^{\circ} 00^{\prime} \mathrm{O}\right)$, Puñihuil (41 $\left.{ }^{\circ} 59^{\prime} \mathrm{S}, 7^{\circ} 03^{\prime} \mathrm{O}\right)$, Duhatao ( $\left.41^{\circ} 59^{\prime} \mathrm{S}, 74^{\circ} 03^{\prime} \mathrm{O}\right)$ e Isla Metalqui ( $\left.42^{\circ} 11^{\prime} \mathrm{S}, 74^{\circ} 08^{\prime} \mathrm{O}\right)$.

Entre octubre y diciembre 2012, en cada sitio se evaluó la riqueza, abundancia y composición de especies macrobentónicas, usando 12 cuadrantes de $50 \times 50 \mathrm{~cm}$ distribuidos en 3 transectos perpendiculares a la línea de costa, y en 4 niveles mareales por transecto dispuestos en forma equidistante entre los límites de las mareas alta y baja, referidos como superior, medio-superior, medio-inferior e inferior. En cada cuadrante se registró la densidad de las especies móviles y la cobertura de las especies sésiles en 25 cuadros, recolectando muestras (fijadas en formalina 10\%) de los organismos no identificados para su determinación posterior en el laboratorio. Excepcionalmente, debido a la dificultad para determinar el número e identidad de sus especies, el gremio de algas coralinas incrustantes fue contabilizado como un solo ítem y tratado como una especie para efectos prácticos.

\section{Carácter biogeográfico y anÁlisis Comunitario}

La identidad y el estatus biogeográfico de las especies encontradas en los sitios de muestreo se evaluó revisando literatura seleccionada para invertebrados (Brattström \& Johanssen 1983, Valdovinos 1999, Lancellotti \& Vásquez 2000, Häussermann \& Försterra 2009) y algas (Etcheverry 1964, Santelices 1980, Ramírez \& Santelices 1981, Hoffmann \& Santelices 1997, Meneses \& Santelices 2000), determinando: (a) su rango latitudinal de distribución en Chile, (b) la existencia de registros previos para el área de estudio, y (c) las unidades biogeográficas (sensu Camus 2001) en las que estaban presentes, incluyendo la mitad sur de la Provincia Peruana templada-cálida ( 18-30/33 ${ }^{\circ}$ S), la Provincia Magallánica templada-fría $\left(\sim 42-56^{\circ} \mathrm{S}\right)$, y la zona de transición entre ambas provincias (Área Intermedia; 30/33-42 $\mathrm{S}$ ).

Para el análisis comunitario de la macrobiota intermareal se calcularon matrices de similitud usando el índice de Bray-Curtis (Bray \& Curtis 1957), a fin de evaluar la diversidad (riqueza + abundancia) de especies sésiles y móviles por separado, previa transformación de sus valores respectivos de cobertura (arcoseno) y densidad $(\log \mathrm{x}+1)$. Un análisis preliminar de las especies sésiles y móviles y del conjuntototal de especies usando el índice binario de Jaccard mostró resultados altamente redundantes con los obtenidos con Bray-Curtis, por lo que sólo se presentan estos últimos. La variación espacial de las especies sésiles y móviles fue evaluada para cada matriz mediante PERMANOVA (Anderson 2001, McArdle \& Anderson 2001) usando 4999 permutaciones, analizando los factores sitio ( $\mathrm{n}=$ 5) y nivel mareal $(n=4)$, su interacción, y las diferencias entre sus niveles a través de pruebas pareadas a posteriori. Adicionalmente, usando la matriz de Bray-Curtis se efectuó: (a) un análisis de los puntos de quiebre de la similitud (SIMPER) para determinar las especies con mayor contribución porcentual a la disimilitud observada (Clarke 1993); y (b) una ordenación de escalamiento multidimensional no métrico (nMDS) para explorar gráficamente las diferencias entre sitios y niveles mareales, realizada separadamente para las especies sésiles y móviles. Todos los análisis anteriores se realizaron usando el programa PRIMER v.6.0 (Clarke \& Gorley 2006).

\section{Resultados}

\section{RIQUEZA DE ESPECIES Y AFINIDAD BIOGEOGRÁFICA}

En el área de estudio se registró un número total de 38 especies (Tabla 1) conformado por: (a) 26 macroinvertebrados, con predominancia de moluscos $(20 ; 77,9 \%)$ incluyendo 16 gastrópodos, 3 poliplacóforos y 1 bivalvo, seguidos por crustáceos cirripedios $(3 ; 11,5 \%)$, equinodermos $(2 ; 7,7 \%)$ incluyendo 1 holoturoídeo y 1 asteroídeo, y cnidarios antozoos (1; 3,8\%); (b) 12 macroalgas, incluyendo 5 rodófitas $(41,7 \%)$, 4 feófitas $(33,3 \%)$ y 3 clorófitas $(25,0 \%)$. A escala local, la riqueza de especies varió desde un máximo de 31 en Guabún $\left(41^{\circ} 48^{\prime} \mathrm{S}, 74^{\circ} 01^{\prime} \mathrm{O}\right)$ a un mínimo de 18 en Isla Metalqui $\left(42^{\circ} 11^{\prime} \mathrm{S}, 74^{\circ} 08^{\prime} \mathrm{O}\right)$, con un promedio ( $\pm \mathrm{DE}$ ) de $24,6 \pm 5,6$ especies por sitio.

En términos biogeográficos, los 36 taxa identificados a nivel de especie (Tabla 1) constituyen organismos comunes en la costa de Chile y ninguno estuvo fuera de los límites conocidos de su rango geográfico. Con excepción de Codium 
Tabla 1. Composición taxonómica de la biota sésil y móvil (columnas 4-8, X = presencia) en los sitios de estudio (Gua: Guabún, MB: Mar Brava, Puñ: Puñihuil, Duh: Duhatao, IM: Isla Metalqui), indicando para cada especie; (a) sus taxa superiores (columna 1; CA: Cnidaria Anthozoa, MP: Mollusca Polyplacophora, M B: Mollusca Bivalvia, MG: Mollusca Gastropoda, CC: Crustacea Cirripedia, EH: Echinodermata Holothuroidea, EA: Echinodermata Asteroidea, C: Chlorophyta, R: Rhodophyta, P: Phaeophyta), (b) su rango latitudinal de distribución en la costa de Chile (columna 2), y (c) su ocurrencia en 3 unidades biogeográficas mayores (sensu Camus 2001) presentes en la costa chilena (columna 3; P: Provincia Peruana, I: Área Intermedia - zona de transición, M: Provincia Magallánica) / Taxonomic composition of the sessile and mobile biota (columns 4-8, X= presence) found at the study sites (Gua: Guabún, MB: Mar Brava, Puñ: Puñihuil, Duh: Duhatao, IM: Isla Metalqui). For each species it is indicated: (a) higher taxa (column 1; CA: Cnidaria Anthozoa, MP: Mollusca Polyplacophora, MB: Mollusca Bivalvia, MG: Mollusca Gastropoda, CC: Crustacea Cirripedia, EH: Echinodermata Holothuroidea, EA: Echinodermata Asteroidea, C: Chlorophyta, R: Rhodophyta, P: Phaeophyta), (b) latitudinal distributional range on the coast of Chile (column 2), and (c) occurrence in each of the 3 major biogeographic units (after Camus 2001) present on the Chilean coast (column 3; P: Peruvian Province, I: Intermediate Area - transition zone, M: Magellanic Province)

\begin{tabular}{|c|c|c|c|c|c|c|c|c|}
\hline Especie & Taxa & $\begin{array}{l}\text { Rango geográfico } \\
\left({ }^{\circ} \mathrm{S}\right)(\text { en Chile) }\end{array}$ & $\begin{array}{c}\text { Unidad } \\
\text { biogeográfica }\end{array}$ & Gua & MB & Puñ & Duh & IM \\
\hline \multicolumn{9}{|l|}{ Biota sésil } \\
\hline Perumytilus purpuratus Lamarck & $\mathrm{MB}$ & $18-56$ & $P-I-M$ & $\mathrm{x}$ & $\mathrm{x}$ & $\mathrm{x}$ & $\mathrm{x}$ & $\mathrm{X}$ \\
\hline Jehlius cirratus Darwin & $\mathrm{CC}$ & $18-43$ & $P-I-M$ & $\mathrm{x}$ & $\mathrm{x}$ & $\mathrm{X}$ & $\mathrm{x}$ & $\mathrm{X}$ \\
\hline Notochthamalus scabrosus Darwin & $\mathrm{CC}$ & $18-55$ & $P-I-M$ & $\mathrm{X}$ & $\mathrm{X}$ & $\mathrm{X}$ & $\mathrm{X}$ & \\
\hline Balanus laevis Brugière & $\mathrm{CC}$ & $18-56$ & $P-I-M$ & $\mathrm{X}$ & & & & \\
\hline Ulva sp. & $\mathrm{C}$ & & & $\mathrm{x}$ & $\mathrm{x}$ & $\mathrm{x}$ & $\mathrm{x}$ & \\
\hline Ulva intestinalis Linnaeus & $\mathrm{C}$ & $21-56$ & $P-I-M$ & $\mathrm{X}$ & $\mathrm{x}$ & & & $\mathrm{X}$ \\
\hline Codium dimorphum Svedelius & $\mathrm{C}$ & $21-54$ & $P-I-M$ & & $\mathrm{X}$ & $\mathrm{x}$ & $\mathrm{x}$ & \\
\hline $\begin{array}{l}\text { Pyropia orbicularis M. E. Ramírez, L. Contreras Porcia \& M. L. } \\
\text { Guillemin }\end{array}$ & $\mathrm{R}$ & $21-56$ & $P-I-M$ & $\mathrm{x}$ & $\mathrm{X}$ & $\mathrm{x}$ & $\mathrm{x}$ & $\mathrm{X}$ \\
\hline Mazzaella laminarioides (Bory) Fredericq & $\mathrm{R}$ & $26-56$ & $P-I-M$ & $\mathrm{X}$ & $\mathrm{X}$ & $\mathrm{x}$ & $\mathrm{x}$ & \\
\hline Dendrymenia skottsbergii (E.Y. Dawson) & $\mathrm{R}$ & $18-44$ & $P-I-M$ & $\mathrm{x}$ & & & $\mathrm{x}$ & $\mathrm{x}$ \\
\hline coralina incrustante & $\mathrm{R}$ & & & & $\mathrm{X}$ & $\mathrm{x}$ & $\mathrm{x}$ & $\mathrm{x}$ \\
\hline Corallina officinalis var. chilensis (Decaisne) Kützing & $\mathrm{R}$ & $18-54$ & $P-I-M$ & $\mathrm{X}$ & $\mathrm{x}$ & $\mathrm{x}$ & $\mathrm{x}$ & $\mathrm{x}$ \\
\hline Halopteris funicularis (Montagne) Sauvegeau & $\mathrm{P}$ & $31-54$ & $\mathrm{I}-\mathrm{M}$ & $\mathrm{X}$ & $\mathrm{x}$ & & $\mathrm{x}$ & $\mathrm{x}$ \\
\hline Durvillaea antarctica (Chamisso) Hariot & $P$ & $31-56$ & $\mathrm{I}-\mathrm{M}$ & $\mathrm{x}$ & $\mathrm{x}$ & & $\mathrm{X}$ & $\mathrm{X}$ \\
\hline Lessonia spicata Suhr & $P$ & $30-41$ & I & $\mathrm{x}$ & $\mathrm{x}$ & $\mathrm{x}$ & & \\
\hline Macrocystis pyrifera (Linnaeus) C. Agardh & $P$ & $31-56$ & $\mathrm{I}-\mathrm{M}$ & $\mathrm{X}$ & & & & \\
\hline Número de especies de biota sésil & 16 & & & 14 & 13 & 10 & 12 & 9 \\
\hline \multicolumn{9}{|l|}{ Biota móvil } \\
\hline Phymactis clematis Drayton & $\mathrm{CA}$ & $18-56$ & $P-I-M$ & $\mathrm{x}$ & $\mathrm{x}$ & & & \\
\hline Chiton granosus Frembly & MP & $18-43$ & $P-I-M$ & $\mathrm{x}$ & $\mathrm{X}$ & $\mathrm{x}$ & $\mathrm{x}$ & $\mathrm{x}$ \\
\hline Tonicia chilensis Frembly & MP & $18-55$ & $P-I-M$ & $\mathrm{X}$ & $\mathrm{x}$ & $\mathrm{X}$ & $\mathrm{x}$ & $\mathrm{x}$ \\
\hline Tonicia disjuncta Frembly & MP & $33-49$ & $\mathrm{I}-\mathrm{M}$ & $\mathrm{X}$ & $\mathrm{x}$ & $\mathrm{x}$ & $\mathrm{x}$ & \\
\hline Lottia orbignyi Dall & MG & $18-42$ & $P-I-M$ & & $\mathrm{x}$ & & $\mathrm{x}$ & \\
\hline Scurria araucana Orbignyi & MG & $18-42$ & $P-I-M$ & & $\mathrm{X}$ & $\mathrm{x}$ & $\mathrm{x}$ & \\
\hline Scurria ceciliana Orbignyi & MG & $18-54$ & $P-I-M$ & $\mathrm{x}$ & $\mathrm{x}$ & $\mathrm{x}$ & & \\
\hline Scurria variabilis Sowerby & MG & $18-42$ & $P-I-M$ & & $\mathrm{x}$ & & $\mathrm{x}$ & \\
\hline Scurria zebrina Lesson & MG & $31-42$ & $\mathrm{I}-\mathrm{M}$ & $\mathrm{x}$ & $\mathrm{x}$ & $\mathrm{x}$ & $\mathrm{x}$ & $\mathrm{X}$ \\
\hline Nacella magellanica Gmelin & $\mathrm{MG}$ & $42-56$ & M & $\mathrm{X}$ & & & & $\mathrm{x}$ \\
\hline Fissurella crassa Lamarck & MG & $18-44$ & $P-I-M$ & $\mathrm{X}$ & & & & \\
\hline Fissurella limbata Sowerby & MG & $18-44$ & $P-I-M$ & $\mathrm{x}$ & & & & $\mathrm{x}$ \\
\hline Fissurella costata Lesson & MG & $18-44$ & $P-I-M$ & & $\mathrm{x}$ & & & $\mathrm{x}$ \\
\hline Fissurella picta Gmelin & MG & $33-56$ & $\mathrm{I}-\mathrm{M}$ & $\mathrm{X}$ & $\mathrm{x}$ & $\mathrm{X}$ & $\mathrm{x}$ & \\
\hline Siphonaria lessonii Blainville & MG & $18-56$ & $P-I-M$ & $\mathrm{x}$ & $\mathrm{x}$ & $\mathrm{x}$ & $\mathrm{x}$ & \\
\hline Austrolittorina araucana $\mathrm{D}^{\prime}$ Orbygni & MG & $18-46$ & $P-I-M$ & $\mathrm{x}$ & $\mathrm{x}$ & $\mathrm{x}$ & $\mathrm{x}$ & $\mathrm{x}$ \\
\hline Prisogaster niger Wood & MG & $18-54$ & $P-I-M$ & $\mathrm{X}$ & & & & \\
\hline Tegula atra Lesson & MG & $18-54$ & $P-I-M$ & $\mathrm{X}$ & $\mathrm{x}$ & $\mathrm{x}$ & $\mathrm{x}$ & $\mathrm{X}$ \\
\hline Acanthina monodon Pallas & MG & $18-56$ & $P-I-M$ & $\mathrm{x}$ & $\mathrm{X}$ & & & \\
\hline Concholepas concholepas Brugière & MG & $18-55$ & $P-I-M$ & $\mathrm{x}$ & $\mathrm{X}$ & & & $\mathrm{X}$ \\
\hline Athyonidium chilensis Semper & EH & $18-42$ & P-I & & & & $\mathrm{x}$ & \\
\hline Stichaster striatus Müller \& Troschel & EA & $18-54$ & $P-I-M$ & $\mathrm{X}$ & & & $\mathrm{x}$ & \\
\hline Número de especies de biota móvil & 22 & & & 17 & 16 & 10 & 13 & 9 \\
\hline Número total de especies & 38 & & & 31 & 29 & 20 & 25 & 18 \\
\hline
\end{tabular}

Vol. 51, № 1, 2016 
Tabla 2. Abundancia de las especies sésiles por nivel mareal en los sitios de estudio, estimada como porcentaje de cobertura (\%) en cuadrantes de $0,25 \mathrm{~m}^{2}(X=$ presencia). Valores: media ( $\pm 1 \mathrm{DE})$ / Abundance of sessile species by tidal level in the study sites, estimated as percent coverage (\%) in $0.25 \mathrm{~m}^{2}$ quadrats ( $\mathrm{X}=$ presence). Values: mean $( \pm 1 \mathrm{SD})$

\begin{tabular}{|c|c|c|c|c|c|c|}
\hline Nivel mareal & Especie & Guabún & Mar Brava & Puñihuil & Duhatao & $\begin{array}{c}\text { Isla } \\
\text { Metalqui } \\
\end{array}$ \\
\hline \multirow[t]{6}{*}{ Superior } & Perumytilus purpuratus & $25,0(40,9)$ & $3,7(4,2)$ & $13,9(3,9)$ & $20,4(13,7)$ & $1,4(2,0)$ \\
\hline & Jehlius cirratus & $18,5(10,5)$ & $27,8(48,1)$ & $11,1(3,9)$ & $\mathrm{X}$ & $20,8(29,5)$ \\
\hline & Ulva sp. & - & $1,9(3,2)$ & $2,8(3,9)$ & $4,6(8,0)$ & - \\
\hline & Mazzaella laminarioides & - & - & - & $18,5(10,5)$ & - \\
\hline & Pyropia orbicularis & $\mathrm{X}$ & $31,5(25,2)$ & $37,5(2,0)$ & - & $5,6(3,9)$ \\
\hline & coralina incrustante & - & - & - & $8,3(14,4)$ & - \\
\hline \multirow[t]{12}{*}{ Medio-superior } & Perumytilus purpuratus & $4,6(8,0)$ & $13,9(5,6)$ & $29,2(17,7)$ & $1,9(3,2)$ & $40,3(25,5)$ \\
\hline & Jehlius cirratus & $36,1(19,4)$ & $3,7(6,4)$ & $25,0(3,9)$ & $\mathrm{X}$ & $4,2(5,9)$ \\
\hline & Notochthamalus scabrosus & - & $\mathrm{X}$ & $4,2(5,9)$ & $\mathrm{X}$ & - \\
\hline & Ulva sp. & - & - & $5,6(3,9)$ & $10,2(7,0)$ & - \\
\hline & Ulva intestinalis & $\mathrm{X}$ & - & - & - & $4,2(5,9)$ \\
\hline & Codium dimorphum & - & $7,4(12,8)$ & - & - & - \\
\hline & Mazzaella laminarioides & $30,6(17,3)$ & $2,8(4,8)$ & $5,6(7,9)$ & $41,7(19,4)$ & - \\
\hline & Pyropia orbicularis & - & $8,3(4,8)$ & $25,0(35,4)$ & $11,1(9,6)$ & - \\
\hline & Dendrymenia skottsbergii & $\mathrm{X}$ & - & - & $\mathrm{X}$ & - \\
\hline & coralina incrustante & - & $0,9(1,6)$ & $20,8(29,5)$ & $0,9(1,6)$ & $18,1(25,5)$ \\
\hline & Corallina officinalis var. chilensis & - & - & - & $1,9(3,2)$ & - \\
\hline & Halopteris funicularis & $0,9(1,6)$ & - & - & - & $1,4(2,0)$ \\
\hline \multirow[t]{12}{*}{ Medio-inferior } & Perumytilus purpuratus & $4,6(8,0)$ & $3,7(4,2)$ & $2,8(3,9)$ & - & $4,2(5,9)$ \\
\hline & Jehlius cirratus & $24,1(4,2)$ & - & $8,3(0,0)$ & - & $11,1(15,7)$ \\
\hline & Notochthamalus scabrosus & - & $13,0(7,0)$ & - & $1,9(3,2)$ & - \\
\hline & Ulva sp. & - & $\mathrm{X}$ & $11,1(3,9)$ & $4,6(4,2)$ & - \\
\hline & Codium dimorphum & - & $5,6(9,6)$ & $38,9(31,4)$ & $2,8(4,8)$ & - \\
\hline & Mazzaella laminarioides & $19,4(33,7)$ & $3,7(4,2)$ & $23,6(33,4)$ & $11,1(19,2)$ & - \\
\hline & Pyropia orbicularis & - & $0,9(1,6)$ & - & $15,7(24,9)$ & - \\
\hline & Dendrymenia skottsbergii & $\mathrm{X}$ & - & - & $0,9(1,6)$ & $1,4(2,0)$ \\
\hline & coralina incrustante & - & $20,4(35,3)$ & $26,4(9,8)$ & $\mathrm{x}$ & $61,1(7,9)$ \\
\hline & Corallina officinalis var. chilensis & $\mathrm{X}$ & - & - & $18,5(8,5)$ & $12,5(13,7)$ \\
\hline & Halopteris funicularis & - & $2,8(4,8)$ & - & $\mathrm{X}$ & - \\
\hline & Durvillaea antarctica & - & - & - & $8,3(8,3)$ & $5,6(7,9)$ \\
\hline \multirow[t]{12}{*}{ Inferior } & Notochthamalus scabrosus & $\mathrm{X}$ & $\mathrm{X}$ & - & - & - \\
\hline & Balanus laevis & $5,6(9,6)$ & - & - & - & - \\
\hline & Ulva sp. & $0,9(1,6)$ & $\mathrm{X}$ & $11,1(15,7)$ & $3,8(2,0)$ & - \\
\hline & Ulva intestinalis & - & $\mathrm{X}$ & - & - & - \\
\hline & Codium dimorphum & - & $29,6(37,8)$ & - & $18,5(31,4)$ & - \\
\hline & Mazzaella laminarioides & - & - & - & $3,7(7,9)$ & - \\
\hline & coralina incrustante & - & $25,0(20,0)$ & $100,0(0,0)$ & $7,4(12,8)$ & $56,9(13,7)$ \\
\hline & Corallina officinalis var. chilensis & $75,0(21,0)$ & $13,0(20,1)$ & $77,8(15,7)$ & $25,9(39,3)$ & $45,8(25,5)$ \\
\hline & Halopteris funicularis & $0,9(1,6)$ & - & - & $4,6(2,0)$ & $\mathrm{X}$ \\
\hline & Durvillaea antarctica & $17,6(14,0)$ & $21,3(34,5)$ & - & $12,0(17,7)$ & $29,2(5,9)$ \\
\hline & Lessonia spicata & $17,6(15,8)$ & $36,1(19,2)$ & $25,0(35,4)$ & - & - \\
\hline & Macrocystis pyrifera & $13,0(20,1)$ & - & - & - & - \\
\hline
\end{tabular}


Tabla 3. Abundancia de las especies móviles por nivel mareal en los sitios de estudio, estimada como densidad (individuos $x \mathbf{0 , 2 5} \mathrm{m}^{2} ; X=$ presencia). Valores: media ( $\left.\pm 1 \mathrm{DE}\right)$ / Abundance of mobile species by tidal level in the study sites, estimated as density (individuals $\times 0.25 \mathrm{~m}^{2} ; X=$ presence). Values: mean ( $\pm 1 \mathrm{SD}$ )

\begin{tabular}{|c|c|c|c|c|c|c|}
\hline Nivel mareal & Especie & Guabún & Mar Brava & Puñihuil & Duhatao & Isla Metalqui \\
\hline \multirow[t]{6}{*}{ Superior } & Austrolittorina araucana & $\mathrm{x}$ & $\mathrm{x}$ & $\mathrm{x}$ & $\mathrm{x}$ & $\mathrm{x}$ \\
\hline & Lottia orbignyi & - & $3,0(5,2)$ & - & - & - \\
\hline & Scurria zebrina & - & $2,0(1,7)$ & $0,5(0,7)$ & $0,3(0,6)$ & $3,0(4,2)$ \\
\hline & Scurria araucana & - & $0,3(0,6)$ & - & $4,3(7,5)$ & - \\
\hline & Scurria variabilis & - & $0,3(0,6)$ & - & $8,7(1,5)$ & - \\
\hline & Siphonaria lessonii & $1,7(1,5)$ & $2,0(3,5)$ & $4,5(2,1)$ & $3,3(3,1)$ & - \\
\hline \multirow[t]{7}{*}{ Medio-superior } & Chiton granosus & $15,7(14,2)$ & $2,0(3,5)$ & - & $0,7(1,2)$ & - \\
\hline & Scurria zebrina & $0,3(0,6)$ & $5,7(5,5)$ & $1,0(1,4)$ & - & $2,0(2,8)$ \\
\hline & Scurria ceciliana & $0,7(1,2)$ & $3,3(5,8)$ & $6,5(4,9)$ & - & - \\
\hline & Scurria araucana & - & - & $3,5(4,9)$ & - & - \\
\hline & Fissurella picta & - & - & - & $0,3(0,6)$ & - \\
\hline & Siphonaria lessonii & $3,3(1,2)$ & $0,7(1,2)$ & $4,5(6,4)$ & - & - \\
\hline & Concholepas concholepas & $1,3(2,3)$ & - & - & - & - \\
\hline \multirow[t]{14}{*}{ Medio-inferior } & Phymactis clematis & $\mathrm{x}$ & - & - & - & - \\
\hline & Chiton granosus & $29,7(4,5)$ & $21,7(10,8)$ & $3,5(4,9)$ & $2,0(1,7)$ & - \\
\hline & Tonicia disjuncta & - & - & $1,5(2,1)$ & $1,3(2,3)$ & - \\
\hline & Tonicia chilensis & - & - & - & - & $1,5(2,1)$ \\
\hline & Scurria zebrina & - & $4,7(0,6)$ & $1,0(1,4)$ & - & $3,5(4,9)$ \\
\hline & Scurria ceciliana & - & - & $4,5(2,1)$ & - & - \\
\hline & Scurria araucana & - & - & $2,5(3,5)$ & - & - \\
\hline & Nacella magellanica & - & - & - & - & $1,0(1,4)$ \\
\hline & Fissurella picta & $0,7(1,2)$ & - & $2,0(2,8)$ & $0,7(1,2)$ & - \\
\hline & Fissurella crassa & $0,3(0,6)$ & - & - & - & - \\
\hline & Siphonaria lessonii & $2,0(3,5)$ & $1,0(1,7)$ & - & - & - \\
\hline & Tegula atra & - & $4,7(8,1)$ & $13,0(14,1)$ & - & $0,5(0,7)$ \\
\hline & Acanthina monodon & $0,3(0,6)$ & - & - & - & - \\
\hline & Concholepas concholepas & $0,7(0,6)$ & - & - & - & $1,0(1,4)$ \\
\hline \multirow[t]{17}{*}{ Inferior } & Phymactis clematis & $\mathrm{x}$ & $\mathrm{x}$ & - & - & - \\
\hline & Chiton granosus & $2,3(4,0)$ & $16,7(18,1)$ & $2,0(2,8)$ & $0,3(0,6)$ & $4,0(1,4)$ \\
\hline & Tonicia chilensis & $0,7(0,6)$ & $0,3(0,6)$ & $1,5(0,7)$ & $0,3(0,6)$ & - \\
\hline & Tonicia disjuncta & $1,0(1,0)$ & $1,0(1,7)$ & $2,0(2,8)$ & $0,7(1,2)$ & - \\
\hline & Lottia orbignyi & - & - & - & $1,0(1,7)$ & - \\
\hline & Scurria zebrina & - & $0,3(0,6)$ & - & - & - \\
\hline & Scurria araucana & - & $0,7(1,2)$ & - & - & - \\
\hline & Nacella magellanica & $0,3(0,6)$ & - & - & - & $3,0(4,2)$ \\
\hline & Fissurella picta & $0,7(0,6)$ & $1,3(1,5)$ & $4,0(1,4)$ & $2,3(2,1)$ & - \\
\hline & Fissurella costata & - & $0,7(0,6)$ & - & - & $3,5(2,1)$ \\
\hline & Fissurella limbata & $0,3(0,6)$ & - & - & - & $3,0(4,2)$ \\
\hline & Prisogaster niger & $1,3(2,3)$ & - & - & - & - \\
\hline & Tegula atra & $0,3(0,6)$ & $10,0(17,3)$ & $12,0(8,5)$ & $1,0(1,0)$ & $6,0(8,5)$ \\
\hline & Acanthina monodon & $0,7(1,2)$ & $0,3(0,6)$ & - & - & - \\
\hline & Concholepas concholepas & $0,3(0,6)$ & $0,3(0,6)$ & - & - & $2,5(2,1)$ \\
\hline & Athyonidium chilensis & - & - & - & $2,8(5,9)$ & - \\
\hline & Stichaster striatus & $0,7(1,2)$ & - & - & $0,3(0,6)$ & - \\
\hline
\end{tabular}


dimorphum y Mazzaella laminarioides, catalogadas como especies endémicas de la costa de Chile, la gran mayoría de las algas e invertebrados presenta amplia distribución latitudinal en Chile pero su rango también se extiende hacia áreas vecinas (principalmentePerú, Antártica, islas subantárticas, y Argentina). De las 36 especies identificadas, 27 (75\%) ocurren en las 3 unidades biogeográficas en Chile, $7(19,4 \%)$ en 2 unidades (6 de ellas en el Área Intermedia y Provincia Magallánica), y sólo $2(5,6 \%)$ en una unidad (Lessonia spicata en el Área Intermedia y Nacella magellanica en la Provincia Magallánica) (Tabla 1). En este contexto, la biota dominante del noroeste de la IGChiloé no presentó ningún rasgo indicativo de un remplazo biogeográfico, y además de ser distinta de la biota que caracteriza la zona de archipiélagos de la Provincia Magallánica, fue virtualmente indistinguible de la encontrada en la mayoría de las comunidades de Chile central.

\section{Dominancia DE ESPECIES}

Considerando los sitios en conjunto, los organismos sésiles y móviles más abundantes y característicos del patrón vertical de dominancia fueron (véase Tablas 2 y 3): (a) nivel superior: Jehlius cirratus, Perumytilus purpuratus, Pyropia orbicularis, Austrolittorina araucana, Scurria zebrina y Siphonaria lessonii; (b) nivel medio (medio-superior + medioinferior): J. cirratus, P. purpuratus, Mazzaella laminarioides, Chiton granosus y $S$. zebrina; (c) nivel inferior: algas coralinas incrustantes, Corallina officinalis var. chilensis (en adelante C. officinalis), Lessonia spicata, Durvillaea antarctica, $C$. granosus, Fisurella picta y Tegula atra. Las únicas especies importantes en más de un nivel mareal fueron $J$. cirratus, $P$. purpuratus, y $S$. zebrina en las zonas superior y media, y $C$. granosus en los niveles medio e inferior.

\section{RIQUEZA Y DOMINANCIA DE ESPECIES POR SITIO}

La Figura 2 muestra una zonación esquematizada del intermareal rocoso en cada sitio de estudio, en correspondencia con las descripciones indicadas a continuación.

\section{GuABúN}

En Guabún la riqueza de especies tanto sésiles como móviles incrementó notoriamente desde el nivel superior hacia el nivel inferior, este último con 12 especies exclusivas, 5 sésiles y 7 móviles (Fig. 3). La zonación vertical del intermareal de Guabún estuvo dominada por los siguientes organismos sésiles y móviles (Tablas 2 y 3): (a) nivel superior: P. purpuratus, J. cirratus, $A$. araucana y $S$. lessonii; (b) nivel medio-superior: J. cirratus, $M$. laminarioides, C. granosus, S. lessonii, S. zebrina y Scurria ceciliana; (c) nivel medio-inferior: J. cirratus, $M$. laminarioides, C. granosus, Fissurella crassa, Acanthina monodon y Concholepas concholepas; (d) nivel inferior: $C$. officinalis, D. antarctica, L. spicata, Tonicia chilensis, Tonicia disjuncta, F. picta, Prisogaster niger, $C$. concholepas, Phymactis clematis y Stichaster striatus.

\section{Mar Brava}

En forma similar a Guabún, la riqueza de especies sésiles y móviles en Mar Brava aumentó desde el nivel superior hacia el nivel inferior el cual tuvo 10 especies exclusivas, 4 sésiles y 6 móviles (Fig. 3), aunque las especies móviles estuvieron poco representadas en los niveles medios. Las especies sésiles y móviles que caracterizaron la zonación vertical fueron (Tablas 2 y 3): (a) nivel superior: J. cirratus, P. orbicularis, A. araucana, $S$. lessonii, Lottia orbignyi y $S$. zebrina; (b) nivel medio-superior: P. purpuratus, P. orbicularis, Codium dimorphum, S. zebrina, S. ceciliana y C. granosus; (c) nivel medio-inferior: Notochthamalus scabrosus, coralinas incrustantes, C. granosus, S. zebrina y T. atra; (d) nivel inferior: $C$. dimorphum, algas coralinas incrustantes, $C$. officinalis, L. spicata, D. antarctica, C. granosus, $T$. disjuncta, Scurria araucana, F. picta, T. atra, C. concholepas y P. clematis.

\section{PuñIHuII}

A diferencia de Guabún y Mar Brava, en Puñihuil las especies sésiles mostraron mayor riqueza en los 2 niveles medios y las móviles en los niveles medio-inferior e inferior, con un bajo número de especies exclusivas de cada nivel (2 en el nivel inferior y 1 en los demás niveles) (Fig. 3). Los organismos que caracterizaron la zonación de Puñihuil fueron (Tablas 2 y 3): (a) nivel superior: $P$. orbicularis, $P$. purpuratus, J. cirratus, $A$. araucana y $S$. lessonii; (b) nivel medio-superior: $P$. purpuratus, J. cirratus, P. orbicularis, coralinas incrustantes, S. lessonii, S. ceciliana y $S$. araucana; (c) nivel medio-inferior: C. dimorphum, M. laminarioides, coralinas incrustantes, $T$. atra, C. granosus, T. disjuncta, $S$. ceciliana y $S$. araucana; (d) nivel inferior: L. spicata, coralinas incrustantes, $C$. officinalis, T. atra, F. picta, T. chilensis y T. disjuncta.

\section{Duhatao}

La riqueza de especies en Duhatao aumentó desde el nivel superior al inferior, pero mostró un patrón vertical opuesto entre las especies sésiles, con mayor riqueza en los dos niveles medios, y las móviles, más numerosas en los niveles superior e inferior (Fig. 3). El nivel inferior fue el que concentró mayor número de especies móviles exclusivas (Fig. 3). La especies características de la zonación en Duhatao fueron (Tablas 2 y 3): (a) nivel 

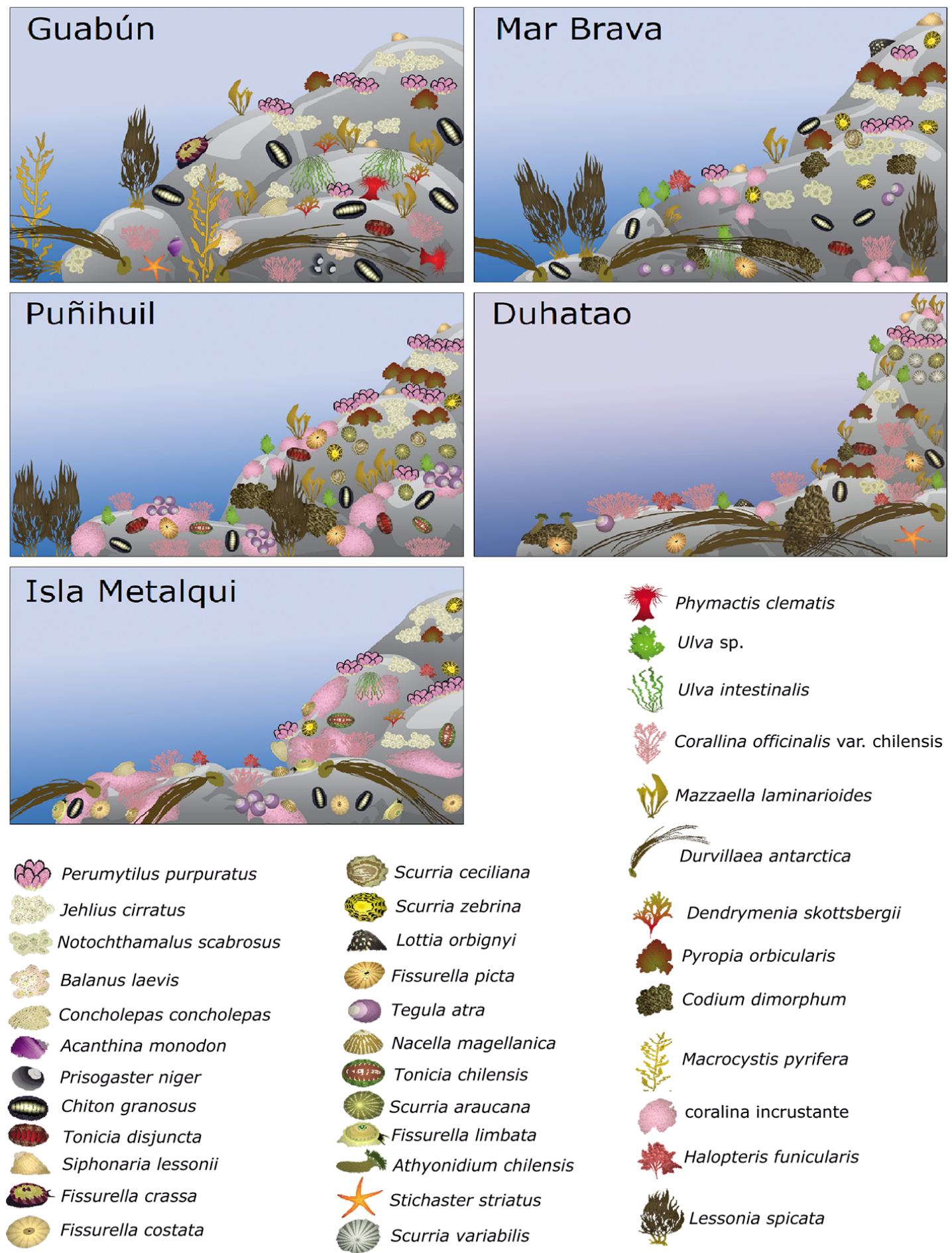

Figura 2. Representación esquemática de los patrones de zonación de la biota intermareal rocosa en 5 localidades de la costa oeste de la Isla Grande de Chiloé / Schematic representation of the zonation patterns of the rocky intertidal biota from 5 localities in the west coast of the Isla Grande de Chiloé 

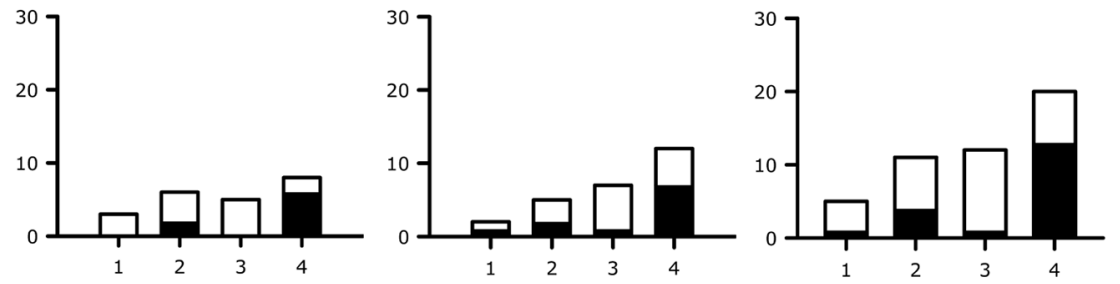

Guabún
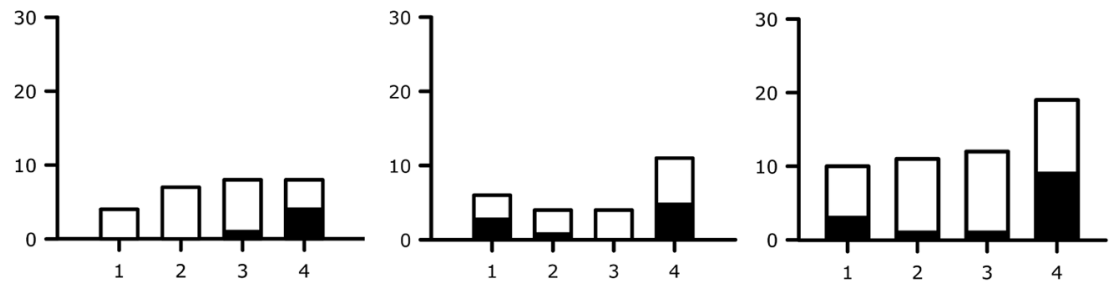

Mar Brava
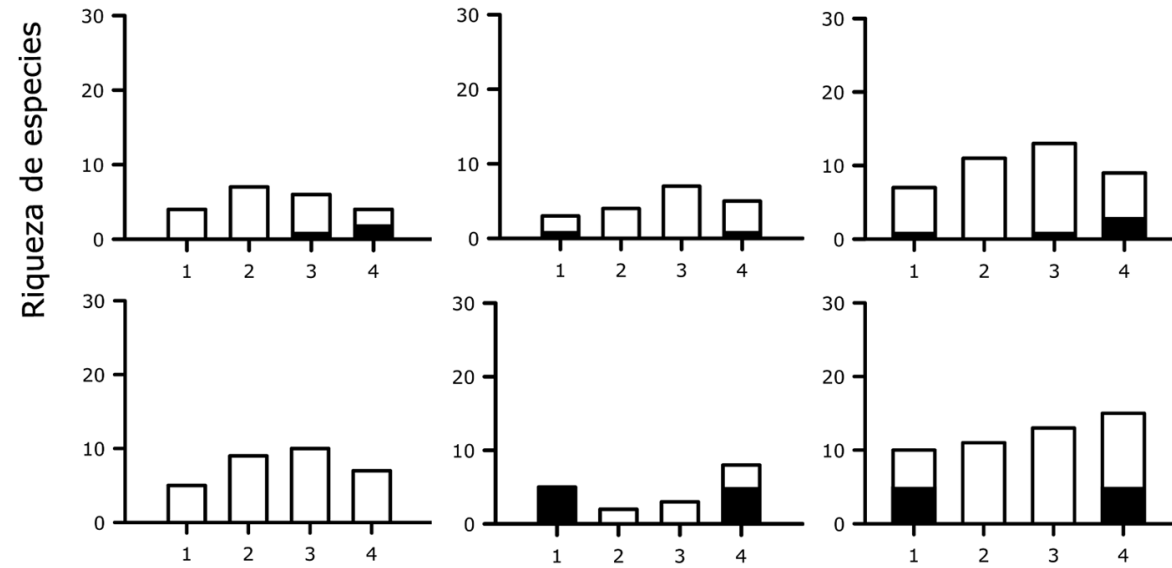

Puñihuil
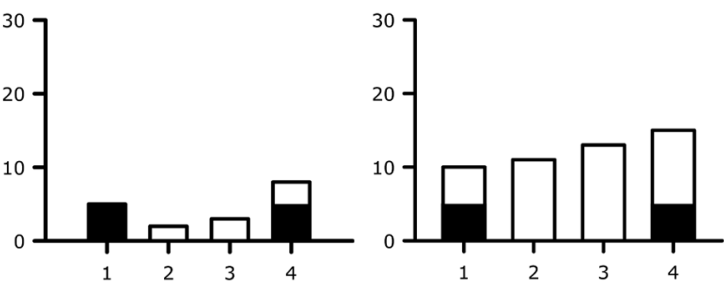

Duhatao
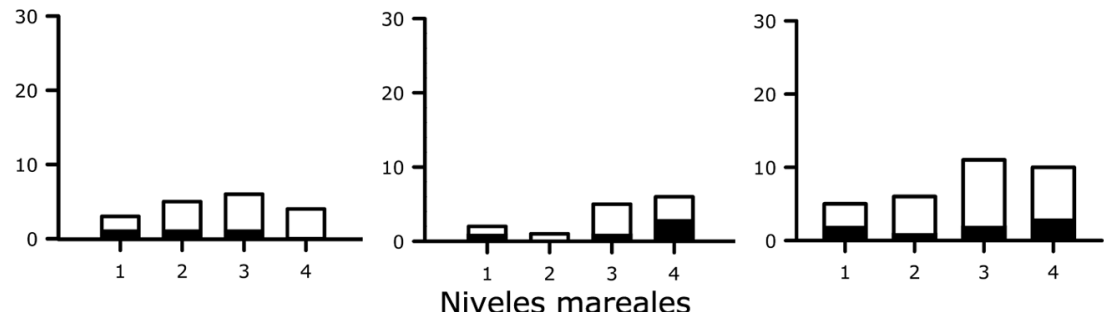

Isla Metalqui

Figura 3. Distribución vertical de la riqueza de especies en los sitios de estudio, estimada para la biota sésil y móvil y para el ensamble completo. Niveles mareales: 1: superior, 2: medio-superior, 3: medio-inferior, 4: inferior. Lasáreas negras de las barras indican el número de especies exclusivas de cada nivel mareal / Vertical distribution of species richness in the study sites, estimated for the sessile and mobile biota and for the whole assemblage. Tidal levels: 1: upper, 2: midupper, 3: mid-lower, 4: lower. The black areas in the bars indicate the number of species exclusive of each tidal level

superior: P. purpuratus, $M$. laminarioides, A. araucana, $S$. lessonii, $S$. variabilis y $S$. araucana; (b) nivel medio-superior: M. laminarioides, $P$. orbicularis, Ulva sp., C. granosus y $F$. picta; (c) nivel medio-inferior: P. orbicularis, $M$. laminarioides, F. picta, C. granosus y T. disjuncta; (d) nivel inferior: C. officinalis, C. dimorphum, D. antarctica, F. picta, T. chilensis, T. disjuncta, Athyonidium chilensis y $S$. striatus. Por otra parte, Duhatao fue el único sitio donde el holoturoídeo
A. chilensis estuvo presente y en alta densidad, debido aparentemente a la abundancia de grietas y pozas intermareales que son los hábitats más propicios para esta especie.

\section{Isla Metalqui}

La riqueza de especies en Isla Metalqui tendió a aumentar hacia el nivel inferior, pero con un máximo en el nivel medio-inferior. Las especies sésiles mostraron mayor riqueza en los dos niveles 
Tabla 4. Análisis de permutaciones (PERM ANOVA). El diseño fue de tipo anidado, considerando los factores: sitio, nivel mareal y la interacción entre sitio-nivel mareal. Los datos se basaron en la similitud de Bray-Curtis, fueron transformados a arcoseno (biota sésil) y $\log x+1$ (biota móvil) y se realizaron 4999 permutaciones / Analysis of permutations (PERMANOVA). The sampling design was nested, considering site, tidal level and the interaction between site-tidal level. Data were transformed to the arcoseno (sessile biota) y log $x+1$ (mobile biota), based in Bray-Curtis similarity analysis, the number of permutations was of 4999

\begin{tabular}{|c|c|c|c|c|c|c|c|c|}
\hline & \multicolumn{4}{|c|}{ Biota sésil } & \multicolumn{4}{|c|}{ Biota móvil } \\
\hline & $d f$ & MS & Pseudo-F & $P$ & $d f$ & MS & Pseudo-F & $P$ \\
\hline Sitio (S) & 4 & 9240 & 8,3901 & 0,0002 & 4 & 9240 & 2,8304 & 0,0002 \\
\hline Nivel mareal $(\mathrm{N})$ & 3 & 16921 & 15,364 & 0,0002 & 3 & 16921 & 4,539 & 0,0002 \\
\hline $\mathrm{S} \times \mathrm{N}$ & 12 & 3106,2 & 2,8205 & 0,0002 & 12 & 3106,2 & 1,2522 & $0,0784(*)$ \\
\hline Residual & 32 & 1101,3 & & & 32 & 1101,3 & & \\
\hline
\end{tabular}

medios, mientras las móviles tendieron a aumentar hacia el nivel inferior (Fig. 3). Los niveles superior, medio-superior y medioinferior tuvieron una especie sésil exclusiva y el nivel inferior dos especies móviles exclusivas (Fig. 3). La zonación en Isla Metalqui estuvo representada primariamente por (Tablas 2 y 3): (a) nivel superior: J. cirratus, A. araucana y S. zebrina; (b) nivel medio-superior: P. purpuratus, coralinas incrustantes, y S. zebrina; (c) nivel medio-inferior: coralinas incrustantes, $C$. officinalis, N. scabrosus, $S$. zebrina, T. chilensis y $C$. concholepas; (d) nivel inferior: coralinas incrustantes, $C$. officinalis, D. antarctica, T. atra, C. granosus, $C$. concholepas, Fissurella costata y otras especies de lapas.

\section{Análisis Comunitario}

En concordancia con las variaciones en los patrones locales de dominancia descritas anteriormente, los resultados de PERMANOVA para la diversidad de especies sésiles mostraron diferencias significativas entre sitios (Pseudo- $\mathrm{F}=8,39 ; P=$ 0,0002 ) y entre niveles mareales (Pseudo-F= 15,36; $P=$ 0,0002) (Tabla 4). No obstante hubo una interacción significativa entre ambos factores (Pseudo-F= 2,82; $P=$ 0,0002) (Tabla 4), debida a que en Guabún y Mar Brava la riqueza de especies aumenta sostenidamente hacia el nivel mareal inferior mientras en los demás sitios esta tendencia existe pero se revierte en el nivel inferior o desde el nivel medio-inferior. Las pruebas pareadas entre sitios mostraron diferencias significativas en cinco de las 10 comparaciones $(0,0096 \leq P \leq$ 0,0388 ) que mostraron a los sitios de Duhatao y Guabún como los más distintivos, contrastando con la alta similitud comparativa entre Mar Brava, Puñihuil e Isla Metalqui. Las diferencias entre niveles mareales fueron significativas en 4 de las 6 comparaciones $(0,0014 \leq P \leq 0,0334)$ que en general reflejaron el contraste entre las zonas extremas del gradiente vertical intermareal (e.g., no hubo diferencia entre los niveles medioinferior y medio-superior). La ordenación gráfica de la diversidad (nMDS; Fig. 4) reflejó con claridad las diferencias a través del gradiente vertical y el carácter comparativamente distintivo de Duhatao y Guabún. El análisis SIMPER mostró que las diferencias detectadas se debieron principalmente a la variación entre sitios en la presencia y abundancia de especies particulares a través del gradiente vertical. Las especies sésiles con mayor contribución individual (> 10\%) a la diferenciación entre sitios en cada nivel mareal, listadas en orden decreciente de importancia individual e indicando su contribución conjunta (porcentaje acumulativo), fueron: (a) nivel superior: J. cirratus, P. orbicularis, P. purpuratus y M. laminarioides con 100\%; (b) nivel medio-superior: M. laminarioides, P. purpuratus, J. cirratus y P. orbicularis con 92,0\%; (c) nivel medio-inferior: J. cirratus, C. officinalis, N. scabrosus y coralinas incrustantes con 78,5\%; (d) nivel inferior: C. officinalis, coralinas incrustantes, L. spicata y D. antarctica con $85,8 \%$.

La diversidad de las especies móviles reveló un patrón mucho más heterogéneo que el de las especies sésiles, reflejado en la ordenación gráfica (nMDS; Fig. 4), mostrando diferencias significativas entre sitios (Pseudo-F=2,83; $P=0,0002$ ) y niveles mareales (Pseudo-F=4,54; $P=0,0002$ ) (Tabla 4) pero en este caso sin interacción entre ambos factores (Pseudo-F= 1,$25 ; P=0,0784$ ) (Tabla 4). Nueve de las 10 comparaciones pareadas entre sitios mostraron diferencias significativas $(0,0002$ $\leq P \leq 0,0164$ ), siendo Mar Brava e Isla Metalqui los únicos sitios estadísticamente similares, mientras que todas las diferencias entre niveles mareales fueron significativas $(0,0002$ $\leq P \leq 0,0368)$. De acuerdo a los resultados del análisis SIMPER, las especies móviles con mayor contribución (> 10\%) a la diferenciación entre sitios por nivel mareal, en orden decreciente de importancia individual e indicando su contribución conjunta (porcentaje acumulativo), fueron: (a) nivel superior: S. lessonii y S. variabilis con 90,3\%; (b) nivel mediosuperior: $C$. granosus, $S$. lessonii, S. zebrina y $S$. ceciliana con $100 \%$; (c) nivel medio-inferior: $C$. granosus y $S$. zebrina con 90,4\%; (d) nivel inferior: F. picta, C. granosus, T. atra y F. costata con $83,0 \%$. 

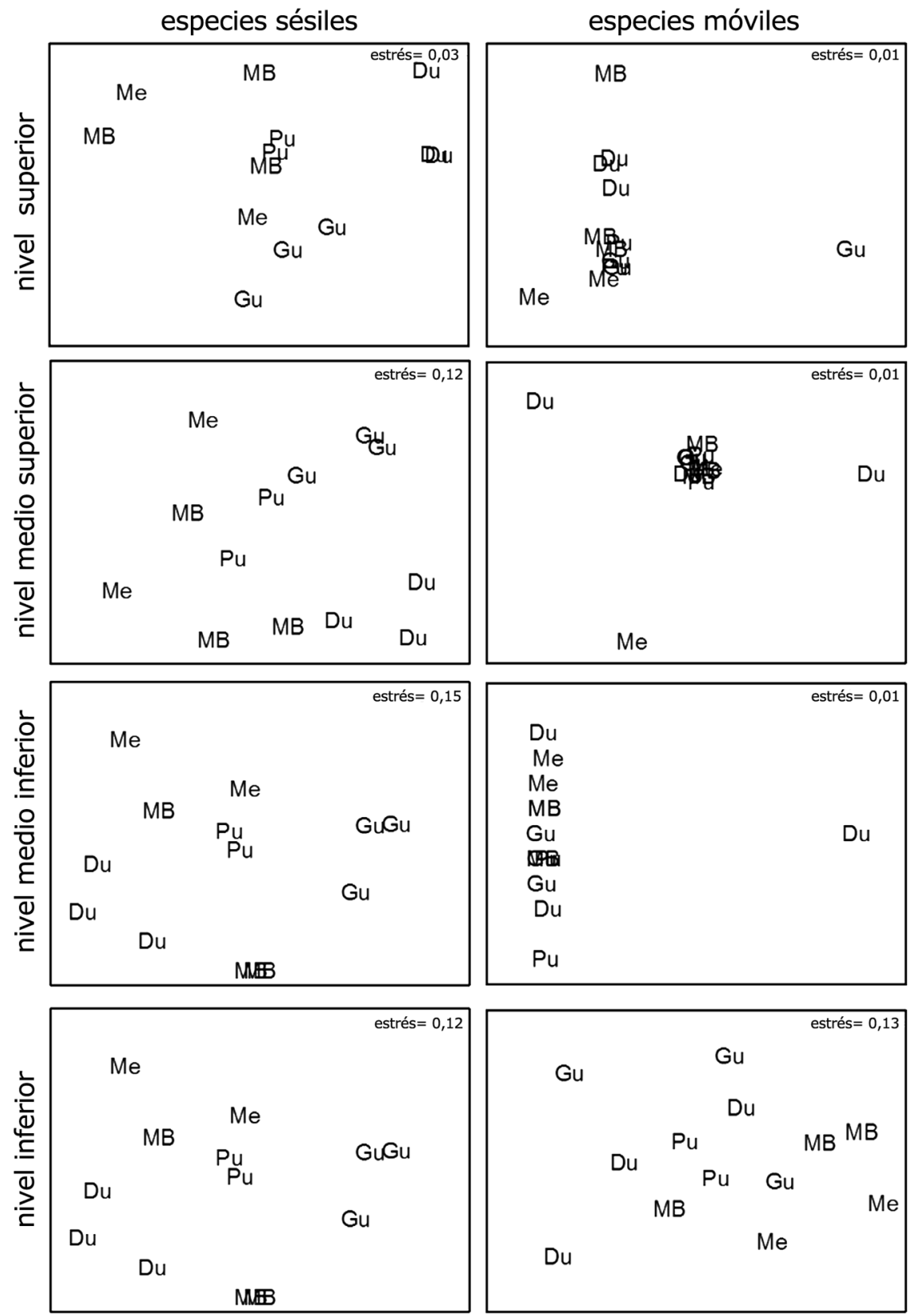

Figura 4. Ordenación multivariada (nMDS) de la diversidad de especies sésiles y móviles en el área de estudio, basada en una matriz de similitud de Bray-Curtis (véase métodos). Las abreviaturas corresponde a los sitios de estudio (Gu: Guabún, MB: Mar Brava, Pu: Puñihuil, Du: Duhatao, Me: Isla Metalqui) y los niveles mareales (superior, medio-superior, medioinferior, inferior) / Multivariate ordination (nMDS) of the diversity of sessile and mobile species in the study area, based on a Bray-Curtis similarity matrix (see methods). The abbreviations correspond to the study sites (Gu: Guabún, MB: Mar Brava, Pu: Puñihuil, Du: Duhatao, Me: Isla Metalqui) and tidal levels (upper, mid-upper, mid-lower, lower) 
Una ordenación nMDS (estrés= 0,12) de la composición taxonómica del ensamble completo mostró un patrón general similar al descrito para las especies sésiles y móviles por separado (Fig. 4), sólo con diferencias menores en la posición de niveles mareales particulares en algunos sitios, por lo cual esta gráfica fue omitida de los resultados.

\section{Discusión}

A nivel ecológico y biogeográfico, el rasgo más importante de las comunidades intermareales rocosas en la costa noroeste expuesta de la IG-Chiloé fue la ausencia de diferencias relevantes con las comunidades en la costa no fragmentada de Chile particularmente entre los $42^{\circ} \mathrm{S}$ y los $30^{\circ} \mathrm{S}$. Si bien esta conclusión no deriva de una hipótesis probada formalmente en este trabajo, tanto los patrones de zonación y dominancia descritos en los resultados como su variación entre sitios son ampliamente concordantes con los documentados en áreas más al norte (e.g., Santelices 1980, Castilla 1981, Broitman et al . 2001, 2011; Thiel et al. 2007). En un contexto más amplio, tanto la estructura de la zonación vertical como los tipos de especies que la caracterizan fueron en general similares a los observados en otras áreas del hemisferio sur, particularmente Australia, Nueva Zelanda y Tasmania (e.g., Guiler 1950, Batham 1956, Womersley \& Edmonds 1958, Stephenson \& Stephenson 1972).

Al comparar la información obtenida con la literatura tradicional y reciente (e.g., Guiler 1959a, b; Stephenson \& Stephenson 1972, Alveal et al. 1973, Viviani 1975, Romo \& Alveal 1977, Santelices 1980, Castilla 1981, Vásquez et al. 1998, Camus \& Andrade 1999, Broitman et al. 2001, 2011; Rivadeneira \& Fernández 2005, Camus 2008), los patrones generales de zonación, dominancia y composición específica en la IG-Chiloé muestran una alta similitud con aquellos ya descritos para las costas de Chile central y norte, con sólo diferencias menores y sitio-dependientes en la dominancia o distribución vertical de especies particulares. A escala local, la similitud se extiende además a los patrones de variación: (a) dentro de sitios, tales como los cambios en dominancia vertical entre cirripedios y mitílidos (e.g., Navarrete \& Castilla 1990), o la predominancia de las especies de patelogastrópodos en zonas con alta abundancia de algas (e.g., Espoz et al. 2004, Aguilera 2011, Aguilera et al. 2013); y (b) entre sitios (aparentemente por sus diferencias relativas en la exposición, configuración y pendiente de los substratos rocosos), como los remplazos locales entre los cirripedios J. cirratus y $N$. scabrosus (e.g., Shinen \& Navarrete 2010), remplazos o dominancia compartida de la zona intermareal inferior entre las macroalgas pardas L. spicata y D. antarctica (e.g., Santelices et al. 1980, Westermeier et al. 1994), o variaciones inversas en la abundancia de algas y moluscos herbívoros en general (e.g., Moreno \& Jaramillo 1983, Jara \& Moreno 1984, Aguilera \& Navarrete 2007).

Por otra parte, una de las pocas diferencias respecto a otras zonas fue la mayor importancia comparativa de algunos invertebrados, particularmente el poliplacóforo C. granosus que es una de las especies más abundantes y frecuentes a través del gradiente vertical en todos los sitios de la IG-Chiloé, lo que podría asociarse a un factor biogeográfico. En la zona intermareal de la costa central y/o norte de Chile, $C$. granosus coexiste y comparte la mayoría de su alimento con varios poliplacóforos de alta relevancia ecológica (Otaíza \& Santelices 1985, Camus et al. 2013), de mayor tamaño corporal y usualmente más abundantes, como Acanthopleura echinata o Enoplochiton niger, que sin embargo no ocurren en la Provincia Magallánica (Sanhueza et al. 2008, Camus et al. 2012). En la IG-Chiloé, en cambio, además de C. granosus los únicos dos poliplacóforos registrados fueron $T$. chilensis y $T$. disjuncta, que son especies de menor tamaño encontradas en baja abundancia y restringidas a la zona intermareal baja. Esto sugiere que la alta importancia de $C$. granosus podría ser una respuesta de compensación de densidad (liberación ecológica) ante la ausencia de otras especies de chitones de gran tamaño y ecológicamente similares (e.g., en hábitos tróficos; véase Fig. 2 en Camus et al. 2013).

Por otro lado, los resultados obtenidos validan en parte algunas descripciones tempranas del área (Knox 1960) que no informaban la localización de las especies, pero además confirman que la composición taxonómica del intermareal rocoso en el Archipiélago de Chiloé presenta una clara diferenciación biogeográfica longitudinal. Esto se evidencia por un fuerte contraste entre las costas este y oeste de la IG-Chiloé, si bien la información disponible no permite evaluar si este patrón es gradual o discreto. No obstante, en la costa oeste de la IGChiloé no se detectaron especies características del mar interior protegido, como el cirripedio Elminius kingii, el mitílido Mytilus chilensis, algunas algas de los géneros Iridaea y Ulva, el decápodo Hemigrapsus crenulatus, o gastrópodos del género Crepidula (Skottsberg 1941, Viviani 1975, Jaramillo 1981, Brattström 1990, Häussermann \& Försterra 2009). La mayoría de estas especies son eurihalinas y/o tienen afinidad por ambientes estuarinos o de baja salinidad (e.g., Arenas 1971, Stotz 1983, Chaparro et al. 2008, Urbina et al. 2010), condiciones que predominan en la zona interior debido a la dilución de las masas de agua por un alto aporte de agua dulce vía precipitación y deshielos terrestres, en interacción con el carácter protegido y la alta amplitud mareal del área, entre otros aspectos (e.g., Pickard 1971, Ahumada et al. 2000, Dávila et al. 2002). Esta combinación de factores favorecería el desarrollo 
de comunidades con creciente carácter mixohalino hacia el interior (Alveal \& Romo 1977), por lo cual es probable que la zonación biogeográfica longitudinal sea gradual, asociada básicamente a un gradiente decreciente de salinidad y exposición al oleaje desde oeste a este.

Los resultados indican por lo tanto la necesidad de revaluar el quiebre biogeográfico definido como el inicio de la Provincia Magallánica hacia el sur, que tradicionalmente se ha situado de forma aproximada en la latitud $42^{\circ} \mathrm{S}$ (e.g., Brattström \& Johanssen 1983, Fernández et al. 2000, Camus 2001, Thiel et al. 2007). Si bien para muchos taxa este quiebre es evidente en el mar interior del Archipiélago de Chiloé, simplemente está ausente en la costa noroeste expuesta al océano Pacífico de la IG-Chiloé, la cual puede considerarse una prolongación hacia el sur de la zona de transición (Área Intermedia) entre las provincias Peruana y Magallánica. Por lo tanto las biotas intermareales rocosas de las costas este y oeste de la IG-Chiloé pertenecerían a 2 unidades biogeográficas distintas, y este patrón habría existido al menos desde el último máximo glacial forzado por las condiciones físico-químicas contrastantes entre ambas costas.

Para comprender este escenario, sin embargo, es necesario determinar si el fenómeno de la presencia de biota del Área Intermedia en la costa noroeste de la IG-Chiloé es sólo local o de mayor extensión espacial, y además si es anterior o posterior a la última glaciación, ante lo cual surgen al menos 3 posibles explicaciones históricas parcialmente excluyentes. La primera es que una biota Magallánica prexistente haya sido remplazada por biota del Área Intermedia en un evento cuya época, duración y causas no son conocidas actualmente, lo cual es básicamente una posibilidad que no puede descartarse debido a la total falta de información al respecto. La segunda, una hipótesis de tipo abductivo (por defecto), es que la biota actual del noroeste de la IG-Chiloé ya existía a fines del Pleistoceno (desde hace al menos 20,000 años AP) y persiste hasta hoy debido a la mayor exposición y salinidad de las costas abiertas directamente al Océano Pacífico. Una predicción simple de esta hipótesis es que la biota del Área Intermedia también debiera estar presente en la costa suroeste de la IG-Chiloé, y eventualmente al sur de Chiloé en otras costas insulares expuestas al Pacífico. La tercera hipótesis, también abductiva, es que la biota del noroeste de la IG-Chiloé constituye una biota relicta de fines del Pleistoceno y que persiste debido a que los glaciares no avanzaron sobre esta área particular en el último máximo glacial (Fig. 1). Por tanto se esperaría que la costa suroeste de la IG-Chiloé (al sur del límite de avance glaciar) albergara una biota distinta de carácter principalmente Magallánico. Si este no fuera el caso, la hipótesis anterior sobre el efecto forzante de factores ambientales adquiriría mayor plausibilidad. Para resolver este escenario se requiere evaluar si la biota del Área Intermedia está presente en costas abiertas al Pacífico al sur de los $42^{\circ} \mathrm{S}$, y disponer de evidencia bioarqueológica crucial para determinar dónde y por cuánto tiempo ocurrió en el pasado.

Complementariamente, esta aparente anomalía biogeográfica en la costa oeste de la IG-Chiloé podría estar vinculada además a otras fuentes de variación que operan en la actualidad. Por ejemplo, análisis de la variación inter-comunitaria a escala geográfica en el Pacífico noreste (Blanchette et al. 2008, Fenberg et al. 2015) y en Chile central (Broitman et al. 2011) muestran que los quiebres espaciales en diversidad en general se corresponden con los principales límites biogeográficos conocidos, pero además que las unidades biogeográficas pueden mostrar una diferenciación interna (estructura espacial) importante, que se manifiesta en la ocurrencia de comunidades particulares o grupos de comunidades con características contrastantes. Dichos estudios sugieren que la variación observada se asociaría a variables físicas (e.g., temperatura superficial del mar, concentración de nutrientes, salinidad) relacionadas principalmente con la variación espacio-temporal de la surgencia, pero también a factores biológicos como diferencias en capacidad de dispersión, limitación del reclutamiento, o variación espacial en el nivel de depredación o herbivoría (véase Blanchette et al. 2008, Broitman et al. 2011, Fenberg et al. 2015). Sin embargo el efecto de estos factores sobre la diversidad intermareal del área de Chiloé aún no se ha establecido.

Todo lo anterior revela la necesidad de actualizar la zonificación biogeográfica latitudinal y longitudinal de la biota intermareal en la zona austral de Chile, a fin de establecer la importancia relativa de los procesos ecológicos e históricos involucrados.

\section{Agradecimientos}

Este estudio fue financiado por el Proyecto 'Línea de Base en Áreas de Alto Valor para la Conservación de la Biodiversidad (AAVC) en la Ecorregión Chiloense', del Ministerio del Medio Ambiente, Chile. Los autores agradecen el apoyo del Dr. Iván F. Rodil (Tvärminne Zoological Station, University of Helsinki, Finlandia) en la realización de los análisis estadísticos (PERMANOVA), la asistencia en terreno de los Biólogos Marinos Emilio Acuña y Jonathan Vergara, el trabajo en laboratorio de Marcia González y los comentarios realizados por 2 evaluadores anónimos.

\section{LITERATURA CITADA}

Abarzúa AM, C Villagrán \& PI Moreno. 2004. Deglacial and postglacial climate history in east-central Isla Grande de Chiloé, southern Chile ( $\left.43^{\circ} \mathrm{S}\right)$. Quaternary Research 62: 49-59. 
Aguilera MA \& SA Navarrete. 2007. Effects of Chiton granosus (Frembly, 1827) and other molluscan grazers on algal succession in wave exposed mid-intertidal rocky shores of central Chile. Journal of Experimental Marine Biology and Ecology 349: 84-98.

Aguilera MA. 2011. The functional roles of herbivores in the rocky intertidal systems in Chile: Areview of food preferences and consumptive effects. Revista Chilena de Historia Natural 84:241-261

Aguilera MA, N Valdivia \& BR Broitman. 2013. Spatial niche differentiation and coexistence at the edge: Cooccurrence distribution patterns in Scurria limpets. Marine Ecology Progress Series 483: 185-198.

Ahumada RB, L Pinto \& PA Camus. 2000. The Chilean coast. In: Sheppard CRC (ed). Seas at the millennium: An environmental analysis, pp. 699-717. Pergamon Press, Oxford.

Alveal K, H Romo \& J Valenzuela. 1973. Consideraciones ecológicas de las regiones de Valparaíso y Magallanes. Revista de Biología Marina y Oceanografía 15: 1-29.

Alveal K \& H Romo. 1977. Estudios de distribución vertical de la biota costera en el Seno Reloncaví - Chile. Gayana, Miscelánea 7: 1-28.

Anderson MJ. 2001. A new method for non-parametric multivariate analysis of variance. Austral Ecology 26:32-46.

Arenas JN. 1971. Distribución de Elminius kingii gray (cirr.) en el estuario del río Valdivia. Studies on Neotropical Fauna and Environment 6(3): 199-206.

Armesto JJ, R Rozzi, C Smith-Ramírez \& MTK Arroyo. 1998. Conservation targets in South American temperate forests. Science 282: 1271-1272.

Arroyo MTK, P Marquet, C Marticorena, J Simonetti, L Cavieres, F Squeo, R Rozzi \& F Mazzardo. 2008. El hotspot chileno, prioridad mundial para la conservación. En: CONAMA (eds). Biodiversidad de Chile, patrimonio y desafíos, pp. 90-93. Ocho Libros Editores, Santiago.

Balech E. 1954. División zoogeográfica del litoral Sudamericano. Revista de Biología Marina 4: 184-195.

Batham EJ. 1956. Ecology of southern New Zealand sheltered rocky shore. Transaction of the Royal Society of New Zealand 84: 447-465.

Blanchette CA, CM Miner, PT Raimondi, D Lohse, KEK Heady \& BR Broitman. 2008. Biogeographical patterns of rocky intertidal communities along the Pacific coast of North America. Journal of Biogeography 35: 1593-1607.

Brattström H. 1990. Intertidal ecology of the northernmost part of the Chilean Archipelago. Sarsia 75: 107-160.

Brattström H \& A Johanssen. 1983. Ecological and regional zoogeography of the marine benthic fauna of Chile. Sarsia 68: 289-339.

Bray JR \& JT Curtis. 1957. An ordination of the upland forest communities of southern Wisconsin. Ecological Monographs 27:325-349.
Broitman B, SA Navarrete, F Smiths \& SD Gaines. 2001. Geographic variation of southeastern Pacific intertidal communities. Marine Ecology Progress Series 224: 21-34.

Broitman BR, F Véliz, T Manzur, EA Wieters, GR Finke, PA Fornes, N Valdivia \& SA Navarrete. 2011. Geographic variation in diversity of wave exposed rocky intertidal communities along central Chile. Revista Chilena de Historia Natural 84: 43-154.

Brown JH \& MV Lomolino. 1998. Biogeography, 698 pp. Sinauer Associates, Sunderland.

Bustamante RH \& GM Branch. 1996. Large scale patterns and trophic structure of southern African rocky shores: the roles of geographic variation and wave exposure. Journal of Biogeography 23: 339-351.

Camus PA. 2001. Biogeografía marina de Chile continental. Revista Chilena de Historia Natural 74: 587-617.

Camus PA. 2008. Diversidad, distribución y abundancia de especies en ensambles intermareales rocosos. Revista de Biología Marina y Oceanografía 43(3): 615-627.

Camus PA \& YN Andrade. 1999. Diversidad de comunidades intermareales rocosas del norte de Chile: El supuesto del enriquecimiento por efecto de la surgencia costera. Revista Chilena de Historia Natural 72: 389-410.

Camus PA, AH Navarrete, AG Sanhueza \& LF Opazo. 2012. Trophic ecology of the chiton Acanthopleura echinata on Chilean rocky shores. Revista Chilena de Historia Natural 85: 123135.

Camus PA, PA Arancibia \& MI Ávila-Thieme. 2013. A trophic characterization of intertidal consumers on Chilean rocky shores. Revista de Biología Marina y Oceanografía 48: 431-450.

Cárdenas J, C Aldea \& C Valdovinos. 2008. Chilean marine mollusca of northern Patagonia collected during the Cimar10 fjords cruise. Gayana 72: 202-240.

Castilla JC. 1979. Características bióticas del Pacífico Sur Oriental, con especial referencia al sector chileno. Revista de la Comisión Permanente del Pacífico Sur 10: 167-182.

Castilla JC. 1981. Perspectivas de investigación en estructura y dinámica de comunidades intermareales rocosas de Chile central. II. Depredadores de alto nivel trófico. Medio Ambiente 5: 190-215.

Castilla JC, SA Navarrete \& J Lubchenco. 1993. Southeastern Pacific coastal environments: main features, large scale perturbations and global climate change. In: Mooney HA, E Fuentes \& B Kronsberg (eds). Contrasts between North and South America, pp. 189-193. Academic Press, New York.

Chaparro OR, YA Montiel, CJ Segura, VM Cubillos, RJ Thompson \& JM Navarro. 2008. The effect of salinity on clearance rate in the suspension-feeding estuarine gastropod Crepipatella dilatata under natural and controlled conditions. Estuarine, Coastal and Shelf Science 76: 861-868. 
Clapperton CM. 1994. The quaternary glaciation of Chile: a review. Revista Chilena de Historia Natural 67: 369-383.

Clarke KR. 1993. Non-parametric multivariate analyses of changes in community structure. Australian Journal of Ecology 18: 117-143.

Clarke KR \& RN Gorley. 2006. PRIMER v6: User Manual/ Tutorial, 192 pp. PRIMER-E, Plymouth.

Crisci JV, MM Cigliano, JJ Morrone \& S Roig-Juñent. 1991. Historical biogeography of southern South America. Systematic Zoology 40: 152-171.

Dahl E. 1960. The cold temperate zone in Chilean seas. Proceedings of the Royal Society of London Series B 152: 631-633.

Dávila P, D Figueroa \& E Müller. 2002. Freshwater input into the coastal ocean and its relation with the salinity distribution of austral Chile $\left(35-55^{\circ} \mathrm{S}\right)$. Continental Shelf Research 22(3): 521-534.

Dell RK. 1971. The marine mollusca of the Royal Society Expedition to Southern Chile, 1958-59. Records of the Dominion Museum 7(17): 155-233.

Dugan JE, E Jaramillo, DM Hubbard, H Contreras \& C Duarte. 2004. Competitive interactions in macroinfaunal animals of exposed sandy beaches. Oecologia 139(4): 630-640.

Espoz C, DR Lindberg, JC Castilla \& BW Simison. 2004. Los patelogastrópodos intermareales de Chile y Perú. Revista Chilena de Historia Natural 77: 257-283.

Etcheverry DH. 1964. Distribución geográfica de las algas del Pacífico Sur Oriental. Boletín del Instituto de Biología Marina, Mar del Plata 9: 17-23.

Fenberg PB, BA Menge, PT Raimondi \& MM Rivadeneira. 2015. Biogeographic structure of the northeastern Pacific rocky intertidal: the role of upwelling and dispersal to drive patterns. Ecography 38: 83-95.

Fernández M, E Jaramillo, PA Marquet, CA Moreno, SA Navarrete, FP Ojeda, CR Valdovinos \& JA Vásquez. 2000. Diversity, ecology and biogeography of Chilean benthic nearshore ecosystems: An overview and needs for conservation. Revista Chilena de Historia Natural 73: 629-662.

Ferro I \& JJ Morrone. 2014. Biogeographical transition zones: a search for conceptual synthesis. Biological Journal of the Linnean Society 113: 1-12.

Glasser NF, KN Jansson, S Harrison \& J Kleman. 2008. The glacial geomorphology and Pleistocene history of South America between $38^{\circ} \mathrm{S}$ and $56^{\circ} \mathrm{S}$. Quaternary Science Reviews 27: 365-390.

Guiler ER. 1950. The intertidal ecology of Tasmania. Papers and Proceedings, Royal Society of Tasmania 1949: 135-201.

Guiler ER. 1959a. Intertidal belt-forming species on the rocky coast of northern Chile. Papers and Proceedings, Royal Society of Tasmania 93: 33-58.
Guiler ER. 1959b. The intertidal ecology of the Montemar area, Chile. Papers and Proceedings, Royal Society of Tasmania 93: 164-183.

Häussermann V \& G Försterrra. 2009. Fauna marina bentónica de la Patagonia Chilena, 1000 pp. Nature in Focus, Santiago.

Hedgpeth J. 1969. Distribution of selected groups of marine invertebrates in waters south of $35^{\circ} \mathrm{S}$ Latitude. In: Bushnell VC \& JW Hedgpeth (eds). Introduction to antarctic zoogeography, pp. 222-230. Antarctic Map folio Series, American Geographical Society, New York.

Hervé F, D Quiroz \& P Duhart. 2009. Aspectos geológicos de la región de los fiordos Chilenos. En: Häussermann V \& G Försterra (eds). Fauna marina bentónica de la Patagonia Chilena, pp. 30-42. Nature in Focus, Santiago.

Heusser CJ, LE Heusser \& A Hauser. 1992. Paleoecology of late quaternary deposits in Chiloé continental, Chile. Revista Chilena de Historia Natural 65: 235-245.

Hoffmann AJ \& B Santelices. 1997. Flora marina de Chile central, 434 pp. Ediciones Universidad Católica de Chile, Santiago.

Hulton NRJ, RS Purves, RD McCulloch, DE Sugden \& MJ Bentley. 2002. The Last Glacial Maximum and deglaciation in southern South America. Quaternary Science Reviews 21:233-241.

Jara F \& CA Moreno. 1984. Herbivory and structure in a midlittoral rocky community: A case in southern Chile. Ecology 65: 28-38.

Jaramillo E. 1981. Ofiuroideos de los archipiélagos de Chiloé y los Chonos. Studies on Neotropical Fauna and Environment 16(3): 113-136.

Jaramillo E, C Duarte \& H Contreras. 2000. Sandy beach macroinfauna from the coast of Ancud, Isla de Chiloé, southern Chile. Revista Chilena de Historia Natural 73: 771-786.

Knox G. 1960. Littoral ecology and biogeography of the southern oceans. Proceedings of the Royal Society of London Series B 152:577-624.

Lancellotti DA \& JA Vásquez. 2000. Zoogeografía de macroinvertebrados bentónicos de la costa de Chile: contribución para la conservación marina. Revista Chilena de Historia Natural 73: 99-129.

Larraín A. 1975. Los equinoideos regulares fósiles y recientes de Chile. Gayana, Zoología 35: 3-147.

Latorre C. 2008. Fluctuaciones climáticas cuaternarias y su impacto sobre la biodiversidad en Chile. En: CONAMA (eds). Biodiversidad de Chile, Patrimonio y desafíos, pp. 60-65. Ocho Libros Editores, Santiago.

Latorre C, PI Moreno, G Vargas, A Maldonado, R VillaMartínez, JJ Armesto, C Villagrán, M Pino, L Nuñez \& M Grosjean. 2007. Late quaternary environments and paleoclimate. In: Moreno T \& W Gibbons (eds). The geology of Chile, pp. 309-328. London Geological Society Press, London. 
McArdle BH \& MJ Anderson. 2001. Fitting multivariate models to community data: a comment on distance-based redundancy analysis. Ecology 82: 290-297.

McLoughlin S. 2001. The breakup history of Gondwana and its impact on pre-Cenozoic floristic provincialism. Australian Journal of Botany 49: 271-300.

Meneses I \& B Santelices. 2000. Una reevaluación de la caracterización fitogeográfica de la costa temperada del Pacífico de Sudamérica. Revista Chilena de Historia Natural 73: 615-623.

Menzies R. 1962. The zoogeography, ecology, and systematics of the Chilean marine isopods. Reports of Lund University Chile Expedition 1948-1949 (42). Lunds Universitets Arsskrift. N.F. Avd. 2. Bd. 57(11): 3-162.

Moreno CA \& E Jaramillo. 1983. The role of grazers in the zonation of intertidal macroalgae of the Chilean coast. Oikos 41:73-76.

Mortensen TH. 1952. Echinoidea and Ophiuroidea. Reports of Lund University Chile expedition 1948-1949 (3). Lunds Universitets Arsskrift. N.F. Avd. 2. Bd. 47(8): 3-22.

Moyano HI. 1983. Southern Pacific Bryozoa: A general view with emphasis on Chilean species. Gayana, Zoología 46: 3-45.

Moyano HI. 1996. Holocene bryozoan links between Australia, New Zealand, southern South America, and Antarctica - a preliminary evaluation. In: Gordon DP, AM Smith \& JA GrantMackie (eds). Bryozoans in space and time, pp. 207-219. National Institute of Water \& Atmospheric Research, Wellington.

Myers N, RA Mittermeier, CG Mittermeier, GAB da Fonseca \& J Kent. 1990. Biodiversity hotspots for conservation priorities. Nature 403: 853-858.

Navarrete SA \& JC Castilla. 1990. Barnacle walls as mediators of intertidal mussel recruitment: effects of patch size on the utilization of space. Marine Ecology Progress Series 68: 113-119.

Ortlieb L. 1995. Paleoclimas cuaternarios en el norte grande de Chile. En: Argollo J \& Ph Mourguiart (eds). Cambios cuaternarios en América del Sur, pp. 225-246. ORSTOM, La Paz.

Ortlieb L, N Guzmán \& M Candía. 1994. Moluscos litorales del Pleistoceno superior en el área de Antofagasta, Chile: primeras determinaciones e indicaciones paleooceanográficas. Estudios Oceanológicos 13: 57-63.

Otaíza RD \& B Santelices. 1985. Vertical distribution of chitons (Mollusca: Polyplacophora) in the rocky intertidal zone of central Chile. Journal of Experimental Marine Biology and Ecology 86: 229-240.

Pawson D. 1964. The holothuroidea collected by the Royal Society Expedition to Southern Chile, 1958-1959. Pacific Science 18(4): 453-470.

Pawson D. 1966. The Echinoidea collected by the Royal Society Expedition to southern Chile, 1958-1959. Pacific Science 20(2): 208-210.
Pickard G. 1971. Some physical oceanographic features of Inlets of Chile. Journal of Fisheries Research Board of Canada 28(8): 1077-1106.

Ramajo L \& C Osorio. 2010. Riqueza, abundancia y patrones espaciales de moluscos desde el Estero Reloncaví (41 $\left.{ }^{\circ} 33^{\prime} \mathrm{S}\right)$ a la Boca del Guafo $\left(43^{\circ} 49^{\prime} S\right)$, sur de Chile. Ciencia y Tecnología del Mar 33(1): 57-65.

Ramírez ME \& B Santelices. 1981. Análisis biogeográfico de la flora de Antofagasta (Norte de Chile). Boletín del Museo de Historia Natural 38: 5-20.

Ricciardi A \& E Bourget. 1999. Global patterns of macroinvertebrate biomass in marine intertidal communities. Marine Ecology Progress Series 185: 21-35.

Rivadeneira MM \& M Fernández. 2005. Shifts in southern endpoints of distribution in rocky intertidal species along the southeastern Pacific coast. Journal of Biogeography 32: 203-209.

Romo H \& K Alveal. 1977. Las comunidades del litoral rocoso de Punta Ventanilla Bahía de Quintero, Chile. Gayana, Miscelánea 6: 1-41.

Rovira J, D Álvarez, K Molt \& D Ortiga. 2008. Áreas protegidas en Chile. En: CONAMA (eds). Biodiversidad de Chile, Patrimonio y Desafíos, pp. 513-559. Ocho Libros Editores, Santiago.

Sanhueza AG, AH Navarrete, LF Opazo \& PA Camus. 2008. Caracterización trófica del placóforo intermareal Enoplochiton niger en el norte de Chile: Variación ambiental y patrones dietarios a nivel local y regional. Revista Chilena de Historia Natural 81: 533-546.

Santelices B. 1980. Phytogeographic characterization of the temperate coast of Pacific South America. Phycologia 19: $1-12$.

Santelices B, JC Castilla, J Cancino \& P Schmiede. 1980. Comparative ecology of Lessonia nigrescens and Durvillaea antarctica (Phaeophyta) in central Chile. Marine Biology 59: 119-132.

Shinen JL \& SA Navarrete. 2010. Coexistence and intertidal zonation of chthamalid barnacles along central Chile: Interference competition or a lottery for space? Journal of Experimental Marine Biology and Ecology 392: 176-187.

Silva N, C Calvete \& H Sievers. 1997. Características oceanográficas físicas y químicas de los canales australes chilenos entre Puerto Montt y Laguna San Rafael (Crucero Cimar-Fiordo 1). Ciencia y Tecnología del Mar 20: 23-106.

Simonetti JA. 1999. Diversity and conservation of terrestrial vertebrates in Mediterranean Chile. Revista Chilena de Historia Natural 72: 493-500.

Skottsberg C. 1941. Communities of marine algae in subantarctic and antarctic waters. Kungliga Svenska Vetenskapsakademiens Handlingar. Tredje Serien 19: 1-92.

Smith DA \& RD Simpson. 2002. Spatial variation in the community structure of intertidal habitats at Macquarie Island (sub-Antarctic). Antarctic Science 14: 374-384. 
Stephenson T \& A Stephenson. 1972. Life between tidemarks on rocky shores, 425 pp. WH Freeman, San Francisco.

Stotz WB. 1983. Características poblacionales y aspectos biológicos del cirripedio Elminius kingii Gray en el estuario del Río Lingue (Mehuín, Chile). Studies on Neotropical Fauna and Environment 18(4): 209-216.

Stuardo J. 1964. Distribución de los moluscos marinos litorales en Latinoamérica. Boletín del Instituto de Biología Marina, Mar del Plata 7: 79-91.

Thiel M, EC Macaya, E Acuña, WE Arntz, H Bastias, K Brokordt, PA Camus, JC Castilla, LR Castro, M Cortés, CP Dumont, R Escribano, M Fernández, JA Gajardo, CF Gaymer, I Gómez, AE González, HE González, PA Haye, JE Illanes, JL Iriarte, DA Lancellotti, G Luna-Jorquera, C Luxoro, PH Manríquez, V Marín, P Muñoz, SA Navarrete, E Pérez, E Poulin, J Sellanes, HH Sepúlveda, W Stotz, F Tala, A Thomas, CA Vargas, JA Vásquez \& A Vega. 2007. The Humboldt Current System of Northern-Central Chile: Oceanographic processes, ecological interactions and socioeconomic feedback. Oceanography and Marine Biology an Annual Review 45: 195-345.

Urbina MA, K Paschke, P Gebauer \& OR Chaparro. 2010. Physiological energetics of the estuarine crab Hemigrapsus crenulatus (Crustacea: Decapoda: Varunidae): responses to different salinity levels. Journal of the Marine Biological Association of the United Kingdom 90: 267-273.

Valdovinos C. 1999. Biodiversidad de moluscos chilenos: Base de datos taxonómica y distribucional. Gayana Zoología 63 : 59-112.

Vásquez JA, PA Camus \& FP Ojeda. 1998. Diversidad, estructura y funcionamiento de ecosistemas litorales rocosos del norte de Chile. Revista Chilena de Historia Natural 71: 479-499.
Villagrán C. 1990. Glacial, Late-Glacial, and Post-Glacial climate and vegetation of the Isla Grande de Chiloé, Southern Chile (41-44 $\mathrm{S})$. Quaternary of South America and Antarctic Peninsula 8: 1-15.

Villagrán C, A León \& FA Roig. 2004. Paleodistribución del alerce y ciprés de las Guaitecas durante períodos interestadiales de la Glaciación Llanquihue: provincias de Llanquihue y Chiloé, Región de Los Lagos, Chile. Revista Geológica de Chile 31(1): 133-151.

Villagrán C \& JJ Armesto. 2005. Fitogeografía histórica de la Cordillera de la Costa de Chile. En: Smith-Ramírez C, J Armesto \& C Valdovinos (eds). Historia, biodiversidad y ecología de los bosques costeros de Chile, pp. 99-116. Editorial Universitaria, Santiago.

Viviani CA. 1975. Las comunidades marinas litorales en el Norte Grande de Chile, 196 pp. Publicación Ocasional del Laboratorio de Ecología Marina, Universidad del Norte, Iquique.

Viviani CA. 1979. Ecogeografía del litoral chileno. Studies on Neotropical Fauna and Environment 14:65-123.

Westermeier R, DG Müller, I Gómez, P Rivera \& H Wenzel. 1994. Population biology of Durvillaea antarctica and Lessonia nigrescens (Phaeophyta) on the rocky shores of southern Chile. Marine Ecology Progress Series 110: 187194.

Womersley HBS \& SJ Edmonds. 1958. A general account of the intertidal ecology of south Australian coasts. Australian Journal of Marine and Freshwater Research 9: 217-262. 